\title{
When Pain Hurts: Nociceptive Stimulation Induces a State of Maladaptive Plasticity and Impairs Recovery after Spinal Cord Injury
}

\author{
James W. Grau, Yung-Jen Huang,, Joel D. Turtle, Misty M. Strain,1, Rajesh C. Miranda, \\ Sandra M. Garraway, and Michelle A. Hook ${ }^{2}$
}

\begin{abstract}
Spinal cord injury (SCI) is often accompanied by other tissue damage (polytrauma) that provides a source of pain (nociceptive) input. Recent findings are reviewed that show SCI places the caudal tissue in a vulnerable state that exaggerates the effects nociceptive stimuli and promotes the development of nociceptive sensitization. Stimulation that is both unpredictable and uncontrollable induces a form of maladaptive plasticity that enhances nociceptive sensitization and impairs spinally mediated learning. In contrast, relational learning induces a form of adaptive plasticity that counters these adverse effects. SCI sets the stage for nociceptive sensitization by disrupting serotonergic (5HT) fibers that quell overexcitation. The loss of $5 \mathrm{HT}$ can enhance neural excitability by reducing membrane-bound $\mathrm{K}^{+}-\mathrm{Cl}^{-}$cotransporter 2 , a cotransporter that regulates the outward flow of $\mathrm{Cl}^{-}$. This increases the intracellular concentration of $\mathrm{Cl}^{-}$, which reduces the hyperpolarizing (inhibitory) effect of gamma-aminobutyric acid. Uncontrollable noxious stimulation also undermines the recovery of locomotor function, and increases behavioral signs of chronic pain, after a contusion injury. Nociceptive stimulation has a greater effect if experienced soon after SCI. This adverse effect has been linked to a downregulation in brain-derived neurotrophic factor and an upregulation in the cytokine, tumor necrosis factor. Noxious input enhances tissue loss at the site of injury by increasing the extent of hemorrhage and apoptotic/pyroptotic cell death. Intrathecal lidocaine blocks nociception-induced hemorrhage, cellular indices of cell death, and its adverse effect on behavioral recovery. Clinical implications are discussed.
\end{abstract}

Keywords: learning and memory; neuronal cell death; neuroplasticity; spinal cord injury

\section{Introduction}

$\mathbf{T}$ HE PRESENT REVIEW EXPLORES how pain affects recovery after spinal cord injury (SCI). We will discuss how the processing of pain (nociceptive) signals within the spinal cord is regulated by learning. These studies demonstrate that uncontrollable/ unpredictable noxious stimulation caudal to SCI engages a lasting (memory-like) effect that enhances nociceptive reactivity and inhibits adaptive plasticity. More important, we show that noxious stimulation soon after a contusion injury increases cell loss at the site of injury, undermines locomotor recovery, and enhances the development of chronic pain. At a cellular level, these effects are related to increased expression of tumor necrosis factor (TNF) and proteins linked to cell death (apoptosis and pyroptosis). We conclude by discussing recent work demonstrating that these adverse effects are enhanced, rather than diminished, by treatment with an opiate analgesic (morphine). As an alternative, we propose that generally inhibiting neural activity with intrathecal lidocaine may have therapeutic value.

\section{Noxious Stimulation Sensitizes Nociceptive Circuits within the Spinal Cord}

Our research builds upon decades of work examining how nociceptive signals are regulated within the spinal cord to modulate both motor reactivity (e.g., withdrawal from a noxious stimulus) and neural activity in ascending pain pathways. (Because the term pain refers to a brain-dependent psychological state, a nonpsychological term [nociception] will be used to describe how spinal circuits operate.) Noxious (potentially tissue damaging) stimuli engage nociceptive fibers that project to the superficial laminae of the spinal cord dorsal horn. While it is acknowledged here that

\footnotetext{
${ }^{1}$ Cellular and Behavioral Neuroscience, Department of Psychology, Texas A\&M University, College Station, Texas.

${ }^{2}$ Department of Neuroscience and Experimental Therapeutics, Texas A\&M Health Science Center, Bryan, Texas.

${ }^{3}$ Department of Physiology, Emory University School of Medicine, Atlanta, Georgia.
} 
peripheral changes can modify the afferent input, ${ }^{1}$ our focus will be on how nociceptive signals are processed within the spinal cord.

Neural projections from the dorsal horn elicit both a motor response and engage ascending circuits that lead to the braindependent perception of pain (Fig. 1A). This nociceptive input is regulated by the brain by descending fibers that can quell neural excitability through both opioid and nonopioid (e.g., serotonergic [5HT]) processes. $^{2}$

The traditional view of pain assumed that the processing of nociceptive signals within the spinal cord was essentially hardwired and therefore immutable. It was recognized that experience (learning) could alter both spinal nociceptive reflexes and nociceptive transmission to the brain, but these effects were ascribed to the brain-dependent regulation of nociceptive circuits through descending fibers. ${ }^{3-5}$ Approximately 20 years ago, a series of discoveries transformed this view, demonstrating that noxious stimulation can induce a lasting alteration within the dorsal horn that sensitizes both motor reactivity and pain (Fig. 1B) ${ }^{6-9}$ Nociceptive sensitization can be induced by the peripheral application of an irritant (e.g., formalin, capsaicin, or complete Freunds adjuvant [cFA]) or a noxious shock that engages C-fibers. At a behavioral level, these treatments can enhance reactivity to mechanical stimulation, a phenomenon that has been related to the allodynia (pain elicited by non-noxious touch) associated with chronic neuropathic pain. ${ }^{7,10,11}$ At a cellular level, nociceptive sensitization is associated with markers of neural excitation within the dorsal horn (e.g., the expression of the transcription factor, $c$-fos, and the activation [phosphorylation] of extracellular signal-regulated kinases (ERKs; e.g., ERK1 and ERK2). ${ }^{8,9}$

Within the brain, learning and memory have been linked to longterm potentiation (LTP), long-term depression (LTD), and $\mathrm{N}$ methyl-D-aspartate receptor (NMDAR)-mediated plasticity. ${ }^{12-14}$ Using electrophysiology, researchers have shown that electrical stimulation can induce both LTD and LTP within the spinal cord and have shown that these effects are blocked by pre-treatment with an NMDAR antagonist. ${ }^{8,15,16}$ Likewise, the peripheral application of the transient receptor potential vanilloid receptor subtype 1 (TRPV1) agonist, capsaicin (the active ingredient in chili peppers), enhances neural excitability in the dorsal horn and ascending nociceptive circuits. ${ }^{7}$ At a cellular level, capsaicin engages $c$-fos and increases the phosphorylation of EKR (pERK). Over the last 20 years, research has shown that the lasting (central) sensitization of spinal nociceptive circuits depends upon signal pathways similar to those involved in brain-dependent learning and memory (Fig. 1C). ${ }^{7,8,17}$ Further, work implicating non-neuronal cells (astrocytes and microglia) in the regulation of brain-dependent plasticity has been paralleled by research showing that these cell types modulate the development of central sensitization within the spinal cord. ${ }^{18,19}$ Indeed, in this arena, it could be argued that work on spinal plasticity has helped to motivate the exploration of how non-neuronal cells regulate plasticity within the brain.

An intriguing feature of nociceptive sensitization is that it often has a diffuse effect on spinal processing. For example, a noxious stimulus applied to a hindleg of a rat can impact behavioral reactivity to stimuli applied to the contralateral limb. ${ }^{20}$ This observation is important because it implies that the experience was likely encoded within a circuit beyond a specific afferent neuron, or the dorsal root ganglion, because such modifications would typically only affect reactivity to stimuli applied to the ipsilateral side.

SCI often leads to the development of chronic pain. ${ }^{21,22}$ In spinally contused rats, symptoms of chronic pain (e.g., enhanced mechanical reactivity [EMR]) typically emerge 14-21 days post- injury. The development of chronic pain post-SCI has been linked to a number of processes known to regulate nociceptive circuits, including the disruption in descending serotonergic (5HT) innervation, dysregulation of non-neuronal cells (gliopathy), and a loss of gamma-aminobutyric acid (GABA)-dependent inhibition ${ }^{19,23}$ The result is an increase in neural excitability within the dorsal horn that enhances both motor responses and, if ascending circuits are intact, nociceptive signals to the brain.

Within the SCI literature, a central question concerns, How SCI leads to chronic pain? We ask a different question, How does acute nociceptive input affect $S C I$ ? What we will show is that nociceptive stimulation caudal to injury can inhibit adaptive plasticity, enhance cell loss at the site of injury, and foster the development of chronic pain. We suggest that this work is clinically relevant because many spinal injuries are accompanied by other forms of tissue damage (polytrauma) that engage nociceptive fibers. If the SCI is severe, peripheral tissue damage caudal to injury may not elicit conscious pain. Nonetheless, the incoming nociceptive signals will provide an afferent barrage that can overexcite nociceptive circuits within the spinal cord, triggering a cascade that impacts the extracellular milieu (e.g., the release of proinflammatory cytokines) and gliopathy. ${ }^{19}$ The result, we will show, is an increase in secondary injury.

While our focus will be on the acute effects of nociceptive stimulation, it is important to note that nociceptive stimulation can also impact spinal function after the injury process has resolved. Potential sources of noxious input include stretching, functional electrical stimulation (FES) to prevent foot drop and to reduce muscle atrophy, and peripheral inflammation/sores. These sources of noxious input can both sensitize nociceptive circuits and disrupt physical rehabilitation ${ }^{24-26}$ A particularly good example of this was recently provided by Bouffard and colleagues, who evaluated the impact of nociceptive stimulation (induced by a capsaicin cream applied to the ankle) on locomotor performance in humans. ${ }^{26} \mathrm{Al}-$ though capsaicin treatment had little immediate impact on locomotor performance, it disrupted the positive transfer (retention) typically observed across days-it impaired motor memory.

We will show that maladaptive plasticity is not an inevitable consequence of noxious stimulation. Rather, how a nociceptive stimulus is processed is regulated by learning. In general, a stimulus that is applied in a controllable/predictable manner has little adverse effect on spinal function and may, in some cases, promote adaptive plasticity. Our work shows that noxious stimulation can have an adverse effect when it is applied in an uncontrollable/unpredictable manner. Of course, this conclusion mirrors decades of work exploring how environmental events impact brain-dependent aversive states and stress. What is perhaps surprising is that the same functional rules govern how stimuli affect neural circuits within the spinal cord and, as we will show, this has relevance to recovery post-SCI.

\section{Spinal Neurons Can Encode Environmental Relations}

Since 1990, our laboratory has been exploring how environmental relations affect spinal nociceptive plasticity. ${ }^{27-29}$ We have shown that spinal neurons can encode the relationship between stimuli (a form of temporal predictability) and that a behavioral response is related to an environmental outcome (a form of behavioral control). Learning about temporal predictability can be studied using a classical (Pavlovian) conditioning paradigm wherein a cue (the conditioned stimulus [CS]) predicts another event (the unconditioned stimulus [US]). ${ }^{30}$ Temporal predictability can also be introduced by presenting a cue at regular (equally spaced) intervals. Behavioral control is studied by introducing a 
A Pain Circuits

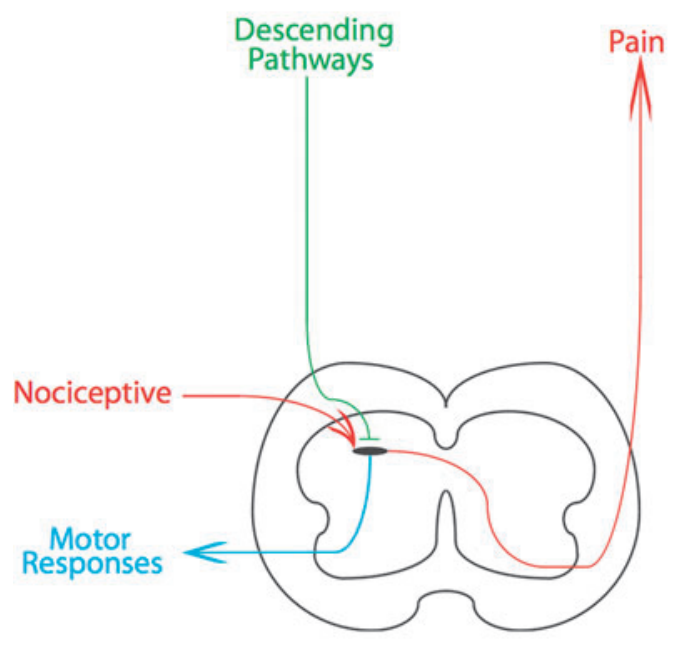

B Nociceptive Signals $\rightarrow$ Sensitization

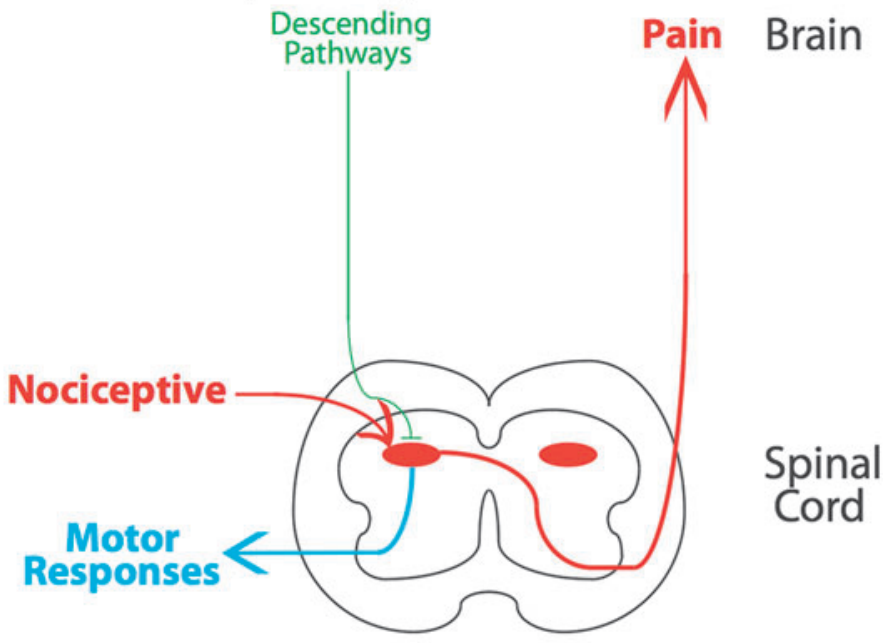

\section{Signal Pathways}

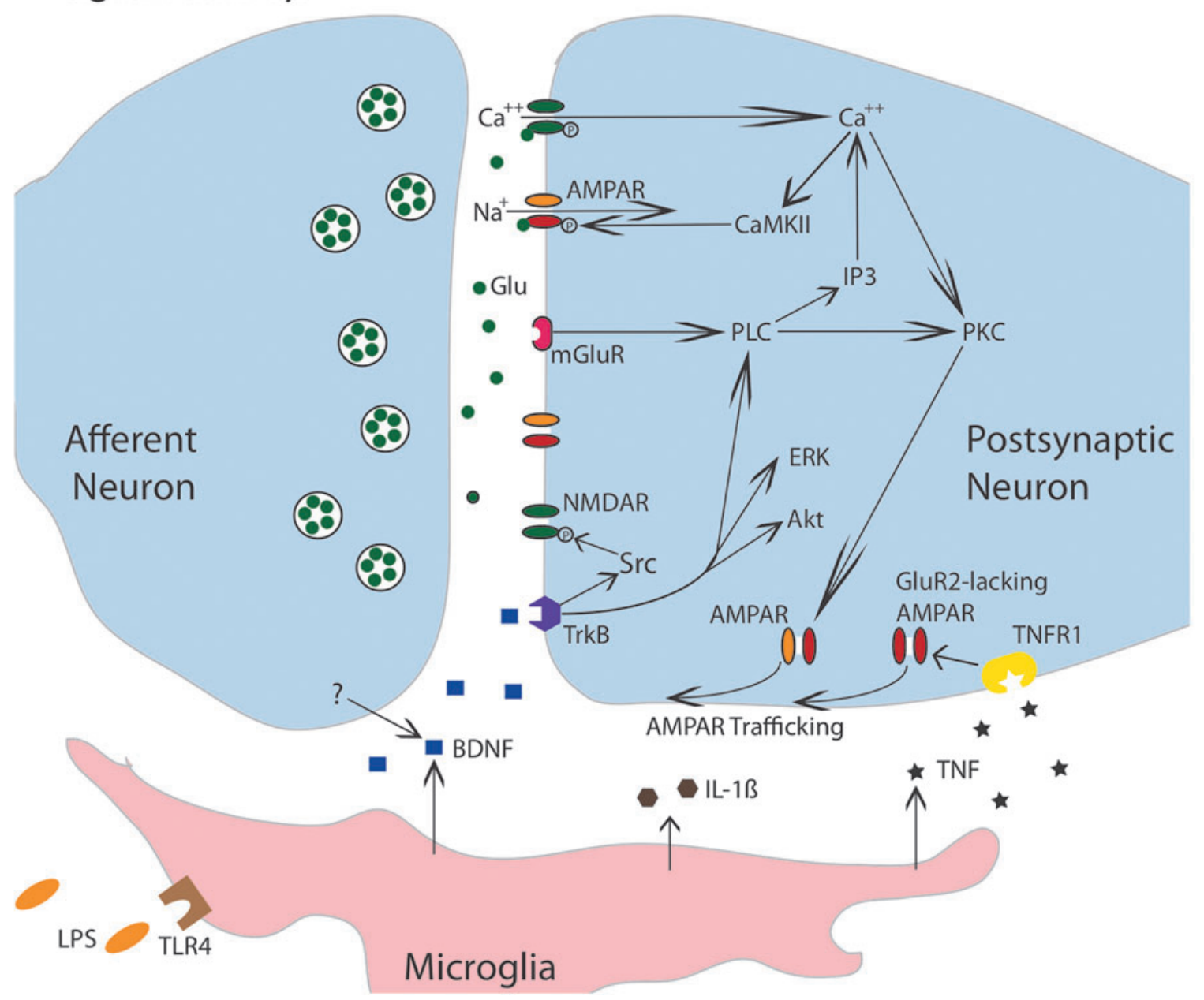

FIG. 1. Mechanisms that contribute to nociceptive sensitization within the spinal cord. (A) A simplified schematic of key nociceptive pathways. Nociceptive afferents project to the spinal dorsal horn and can engage both a motor response (blue) and ascending fibers that relay pain signals to the brain. Descending pathways (green) regulate nociceptive processing within the dorsal horn. (B) Intense nociceptive input can induce a bilateral overexcitation within the dorsal horn that enhances motor reactivity and pain signals to the brain. (C) Nociceptive input engages glutamatergic neurons within the dorsal horn that can produce a lasting modification in neural excitability through signal pathways analogous to those involved in brain-dependent learning and memory. Akt, protein kinase B; AMPAR, $\alpha$-amino-3-hydroxy-5-methyl-4-isoxazolepropionic acid receptor; BDNF, brain-derived neurotrophic factor; CaMKII, calcium/calmodulin activated protein kinase II; ERK, extracellular signal-regulated kinase; GluR2, glutamate receptor 2; IL-1 $\beta$, interleukin-1 beta; IP3, inositol 1,4,5-trisphosphate; mGluR, metabotropic glutamate receptor; NMDAR, N-methyl-D-aspartate receptor; PKC, protein kinase C; PLC, phospholipase C; TrkB, tropomyosin receptor kinase B; TNF, tumor necrosis factor; TNFR1, TNF receptor 1 . 
relationship between a particular response (R) and an environmental outcome (O; aka, the reinforcer). For example, a behavioral contingency can be introduced by applying a noxious shock to the hindlimb of a rat whenever that leg is in an extended position. Over time, learning can elicit an increase in flexion duration that minimizes net shock exposure. Learning about R-O relations is referred to as instrumental conditioning (for a discussion of the distinction between operant and instrumental learning, see $\mathrm{Grau}^{31}$ ). Notice that both Pavlovian and instrumental conditioning are defined by the relation between two events, a stimulus-stimulus (S-S) or R-O relation, respectively. In this way, the learning differs from single stimulus learning (e.g., habituation and sensitization). What we will show is that learning about an environmental relation affects nociceptive sensitization. (We use relation rather than association because the latter term has mechanistic implications that may not apply to spinal learning. ${ }^{32}$ )

\section{Spinal neurons are sensitive to response-outcome relations}

Twenty years ago, it was generally accepted that spinal neurons are sensitive to Pavlovian relations. ${ }^{33,34}$ What was not clear is whether the brain was needed to learn about response-outcome (R-O) relations. ${ }^{27}$ While there were some positive reports, ${ }^{35,36}$ the results had been dismissed on the basis of methodological flaws. ${ }^{37,38} \mathrm{Re}-$ cognizing that instrumental conditioning was relevant to physical therapy, ${ }^{39}$ we worked to develop a paradigm that would address past criticisms. ${ }^{40}$ Our work shows that spinal neurons can support a simple form of instrumental conditioning and provides a useful assay for assessing how experimental treatments affect spinal function.

In order to demonstrate learning without input from the brain, the spinal cord was completely transected at the second thoracic (T2) vertebra. A day after surgery, animals are placed in opaque plastic tubes that provide access to the hindlimbs. Leg position (the $\mathrm{R}$ ) is monitored by means of a contact electrode that is taped to the base of the rat's paw. When the leg is in a relaxed position, the rod touches the underlying salt solution, which completes a circuit monitored by a computer. Shock (the $\mathrm{O}$ ) is applied to the tibialis anterior muscle through a pair of electrodes and shock intensity is adjusted to elicit a flexion force of $0.4-0.6 \mathrm{~N}$. One group of rats (Master) then receives shock whenever the leg is extended. Animals in a second group (Yoke) are experimentally coupled to the master rats. Each animal in the yoked condition receives shock at the same time as its master partner, equating both the duration and distribution of shock exposure. Rats are typically given $30 \mathrm{~min}$ of training and learning is quantified by calculating average flexion duration in 1-min time bins. On this measure, a rat that exhibits a response duration of $60 \mathrm{sec}$ is maintaining its leg in a flexed position for the entire time bin. Conversely, response durations near zero indicate poor learning.

We find that (master) rats given controllable shock exhibit a progressive increase in flexion duration that reduces net shock exposure. ${ }^{40}$ Rats that receive shock independent of leg position (yoked) do not exhibit an increase in flexion duration, which implies that the increase in flexion duration observed in the master condition depends upon the $\mathrm{R}_{\text {(leg position) }} \mathrm{O}_{\text {(shock onset) }}$ relation. Fluid height and flexion force are then re-equated and the rats are tested under common conditions with controllable stimulation. Interestingly, the results here are identical irrespective of whether animals are tested on the same (ipsilateral) or opposite (contralateral) limb. In both cases, rats that had previously received controllable stimulation learn more rapidly (positive transfer) than the naïve (un- shocked) controls. ${ }^{41}$ Surprisingly, yoked rats, that had previously received uncontrollable stimulation, fail to learn. ${ }^{42}$ This failure occurs even though these rats exhibit the highest rate of responding; they repeatedly encounter the R-O relation, but it fails to induce an increase in response duration.

Recognizing that the inhibition of plasticity could have clinical implications, we further explored this phenomenon. To do so, we developed a computer program that generated a variable intermittent shock (VIS) schedule that emulated the distribution of shocks produced by a typical master rat. Shocks $(80 \mathrm{~ms})$ are presented approximately $2 \mathrm{sec}$ apart at varying intervals $(0.2-3.8 \mathrm{sec}$; rectangular distribution). Just 6 min of VIS to the leg or tail produces a learning impairment that lasts $48 \mathrm{~h}^{41}$ The implication is that uncontrollable stimulation generally inhibits spinal learning, a learning deficit reminiscent of learned helplessness. ${ }^{43}$

Exposure to controllable stimulation has the opposite effect on learning potential. This was shown by testing animals using a higher response criterion, achieved by adding solution to the underlying container (to submerge the contact electrode by $8 \mathrm{~mm}$ rather than the usual 4). Increasing the response criterion in this manner makes the task so difficult that naïve controls fail to learn. ${ }^{41}$ However, rats that have previously received $30 \mathrm{~min}$ of controllable shock, either to the same or opposite leg, generally learn when tested with a higher response criterion. This suggests that training with controllable stimulation enables adaptive plasticity (for a discussion of how this work relates to the application of FES, see Hook and $\mathrm{Grau}^{44}$ ).

\section{Spinal neurons are sensitive to temporal relations}

Subsequent work revealed that spinal learning impacts nociceptive circuits within the spinal cord and influences the development of central sensitization. ${ }^{45-47}$ As we will see, in the process of exploring these interactions, we uncovered an additional form of spinal learning.

Nociceptive stimuli can engage both myelinated A fibers and unmyelinated $\mathrm{C}$ fibers. To explore the cell types engaged, we attached shock electrodes to the sciatic nerve and varied shock intensity (volts). ${ }^{48}$ Low-intensity shocks engage just A fibers, whereas higher intensities are needed to recruit a robust C-fiber response. Rats were given shock bursts $(80 \mathrm{msec})$ to the sciatic nerve on a variable schedule $(0.2-3.8 \mathrm{sec}$; mean $=2)$. After $6 \mathrm{~min}$ of stimulation, the capacity for instrumental learning was tested by applying response-contingent shock to the contralateral leg. We found that electrical stimulation of the sciatic nerve only induced a learning impairment when shock intensity was set to an intensity that induced a robust $\mathrm{C}$-fiber response (for additional evidence that $\mathrm{C}$ fibers play a critical role, see Baumbauer and colleagies ${ }^{49}$ ).

Another variable known to regulate the development of plasticity is shock frequency. ${ }^{50}$ To explore how this factor influences spinal learning, we made a seemingly subtle change to our stimulation paradigm: Instead of presenting the shocks on a variable schedule, we presented them in a regular (fixed spaced) manner $2 \mathrm{sec}$ apart. Notice that doing this introduces a form of temporal predictability. Because the capacity to time is typically linked to brain mechanisms, ${ }^{51}$ we assumed that this change would not matter. Indeed, when animals received 6 min of shock (180 shocks), both variable and fixed spaced intermittent shock produced a learning impairment. However, when shock number was increased to 900 , only variable shock induced a learning impairment. ${ }^{48,52}$ For rats given fixed spaced shock, continued exposure (an additional 720 stimuli) had a restorative effect that eliminated the learning impairment induced by the initial 180-360 shocks. 
These results imply that spinal neurons can discriminate fixed and variable stimulation - that they have some capacity to track temporal relations. We have investigated the mechanisms that underlie these effects and shown that a central spinal system can abstract regularity across a break in time (savings) even when the locus of stimulation is varied. ${ }^{53,54} \mathrm{We}$ have suggested that regular stimulation has a distinct effect because it entrains a spinal oscillator, possibly related to the central pattern generator (CPG) that drives the tempo of stepping. Behaviorally, the results imply that the impact of nociceptive stimulation is modulated by both temporal predictability and behavioral control. Our work also shows that variable stimulation has a distinct, opponent-like, effect on spinal plasticity. As we have noted, ${ }^{55,56}$ variable noxious input may engage a distinct category of physiological effects because the pattern of neural activity elicited emulates the erratic firing observed in C fibers in response to peripheral inflammation. ${ }^{8,16}$ The implication is that the consequences of afferent input depend upon both the fiber type(s) engaged and the pattern of neural activity.

Our interest in spinal cord plasticity was initially motivated by the question of whether spinal neurons could support some simple forms of learning. The idea that stimulus exposure could induce a lasting reduction (habituation) or increase (sensitization) in neural excitability/motor reactivity was not in doubt. ${ }^{27,57}$ Of greater interest and controversy was whether spinal neurons can encode the relationship between two events and whether this has a lasting (memory-like) effect on spinal function. Our results show that spinal neurons are sensitive to both behavioral (R-O) and temporal relations and that experience with these relations has a lasting effect on how spinal neurons operate.

\section{Relational learning depends upon the $N$-methyl-D- aspartate receptor and engages brain-derived neurotrophic factor}

Naturally, given the discovery of NMDAR-mediated spinal plasticity, ${ }^{15,16}$ we examined whether spinally mediated instrumental learning is affected by pre-treatment within an NMDAR antagonist. ${ }^{46,58}$ Here, and in subsequent experiments, drugs are typically applied to the rostral lumbosacral spinal cord by means of an intrathecal (i.t.) catheter. We found that i.t. application of a competitive ((2R)-amino-5-phosphonovaleric acid; APV), or noncompetitive (MK-801), NMDAR antagonist disrupted instrumental learning in a dose-dependent manner. Similarly, pre-treatment with MK-801 disrupts learning about temporal relations. ${ }^{52}$

More recently, we have begun to explore the neurochemical systems that mediate the long-term effects of training. In collaboration with Gómez-Pinilla and colleagues, ${ }^{59}$ we showed that training with controllable stimulation upregulates the expression of a number of genes related to synaptic plasticity, including brain-derived neurotrophic factor (BDNF), calcium/calmodulin activated protein kinase II (CaMKII), and the gene transcription factor, cyclic adenosine monophosphate response element-binding protein (CREB). Exposure to uncontrollable stimulation had the opposite effect.

Controllable stimulation also increases BDNF protein within the lumbosacral tissue. ${ }^{60}$ To explore the functional consequences of this increased expression, we pretreated rats with the BDNF sequestering antibody, TrkB-IgG (tropomyosin receptor kinase B immunoglobulin G). TrkB-IgG had little effect on performance when rats were trained with the usual $(4 \mathrm{~mm})$ response criterion. ${ }^{59}$ We then raised the response criterion (to $8 \mathrm{~mm}$ ). As usual, pretrained vehicle-treated rats were able to learn. Inhibiting BDNF function with TrkB-IgG eliminated the beneficial effect of pre- training. Finally, we applied BDNF before testing with a high response criterion. As expected, naïve rats that received the vehicle could not learn when the response criterion was made more difficult, whereas animals pretreated with BDNF were able to learn. ${ }^{59}$ The results show that training with controllable stimulation induces the expression of BDNF, which enables learning.

In summary, we have shown that neurons within the lumbosacral spinal cord are sensitive to R-O and temporal relations, that controllable stimulation enables learning, and that uncontrollable/unpredictable stimulation inhibits adaptive plasticity. Further, both processes appear to regulate plastic potential independent of whether rats are tested on the pre-trained or opposite limb. As we have discussed elsewhere, ${ }^{61}$ the enabling/inhibition of plasticity appears to be related to the concept of metaplasticity, because the phenomena have a long-term effect, regulate the capacity to change (plasticity), and are reversible. ${ }^{61,62}$

\section{Relational Learning Modulates the Effect of Noxious Stimulation}

\section{Uncontrollable/unpredictable stimulation induces nociceptive sensitization}

Given the neural complexity of the spinal central gray, and evidence of learning in simpler invertebrates, ${ }^{63}$ it is not surprising that spinal neurons can encode the relationship between two events. What seems more surprising is that the absence of a relation engages an active process that undermines future learning. Of course, it is well documented that brain systems are sensitive to whether an event is controllable or uncontrollable and that temporal uncertainty enhances stress. ${ }^{30,64}$ Our work suggests that a lack of control/ predictability also has a unique effect on spinal function, which impedes future learning. As we will see below, uncontrollable stimulation also adversely affects recovery after a contusion injury. Given the clinical importance of this finding, we sought to further characterize how VIS affects spinal function. Our work shows that spinal injury disrupts descending fibers that regulate spinal nociceptive circuits (Fig. 1A), setting the stage for nociceptive sensitization (Fig. 2A).

Our initial hypothesis was that uncontrollable/unpredictable stimulation impairs learning because it inhibits neural activity in nociceptive circuits. Because past studies had shown that nociceptive processes are regulated by opioid peptides, ${ }^{65,66}$ we tested whether the learning impairment induced by VIS could be blocked with an opioid antagonist (naltrexone) ${ }^{67}$ Administration of naltrexone before testing eliminated the learning impairment induced by VIS in spinally transected rats, implying that the expression of the learning impairment is opioid mediated. To examine whether opioids also play a critical role in the induction of the learning impairment, spinally transected rats were given naltrexone before VIS and tested $24 \mathrm{~h}$ later. Blocking opioid receptors at the time of initial shock exposure had no effect. ${ }^{67}$ This pattern of results implies that the mechanisms engaged at the time of VIS exposure, and that underlie the induction of a lasting modification, are not identical to those involved in the expression of the learning impairment at the time of testing; the latter involves an opioid-dependent process whereas the former does not. Further studies have shown that learning is inhibited by a ligand that acts at the kappa opioid receptor. ${ }^{68}$

Reasoning that the inhibition of plasticity could be mediated by GABA, we tested the effect of the GABA type A (GABA-A) receptor antagonist, bicuculline. We found that bicuculline administered before testing blocked the expression of the learning 
A Sensitization After Injury

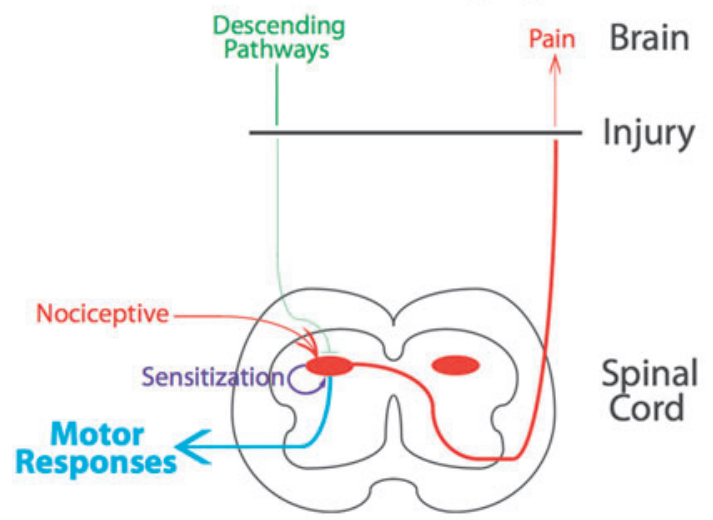

\section{Shock $\rightarrow$ EMR}

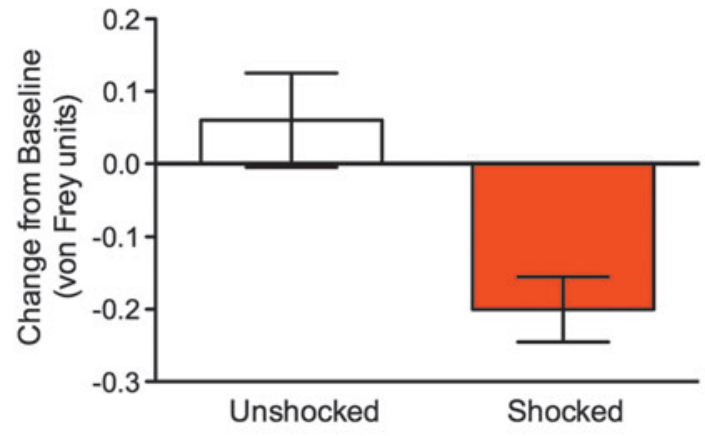

E Learning Inhibits Capsaicin EMR

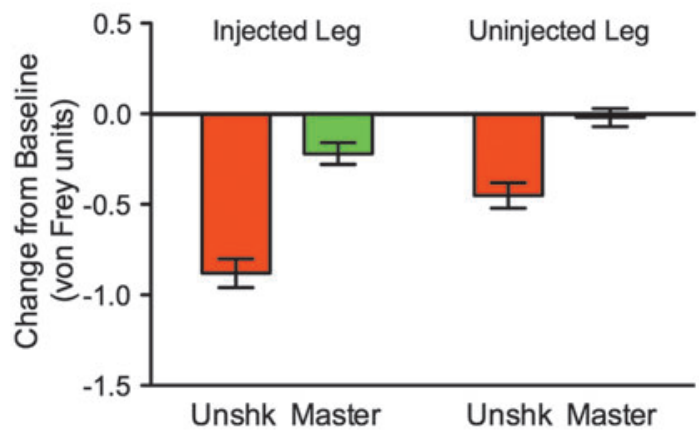

B Learning Inhibits Sensitization

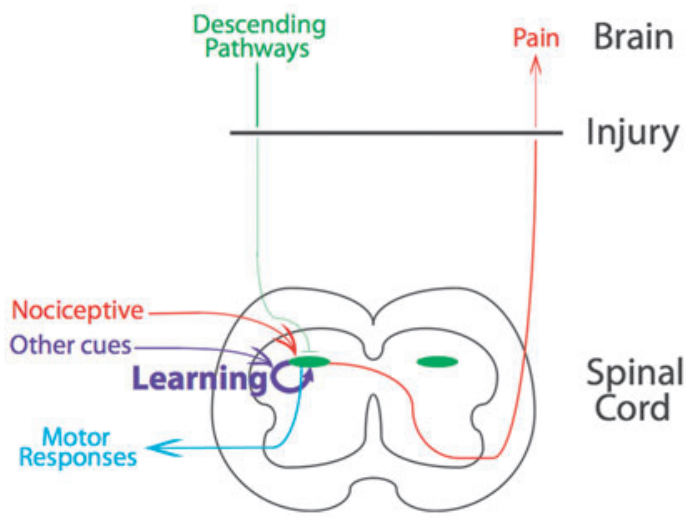

D Capsaicin $\rightarrow$ Learning Deficit

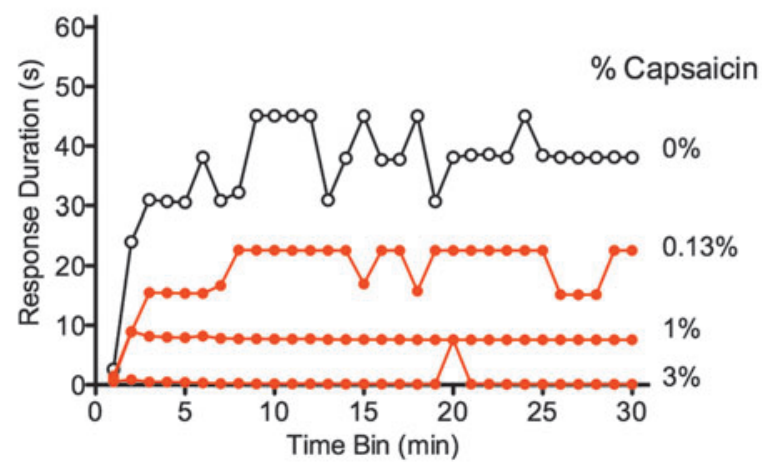

F Prior Learning Blocks the Deficit

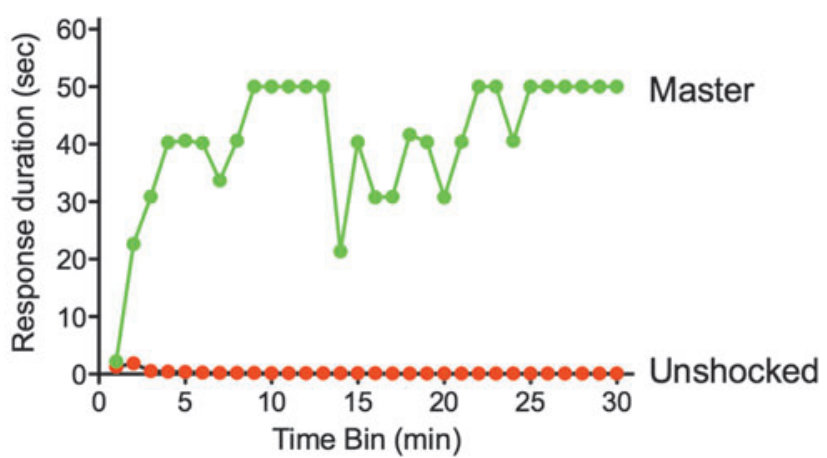

FIG. 2. Induction and regulation of nociceptive sensitization after spinal injury. (A) Spinal injury can disrupt descending fibers that normally quell overexcitation within the dorsal horn, enabling development of central sensitization. (B) Relational learning can attenuate nociceptive sensitization and enable adaptive plasticity. Learning depends upon cues that indicate a nociceptive stimulus occurs at a particular position (the R) or time. (C) Variable intermittent shock (red) enhanced reactivity to mechanical stimulation in spinally transected rats. (D) Application of capsaicin to one hindpaw impaired instrumental learning. Spinally transected rats were tested by applying response-contingent shock to the contralateral hindleg. In the absence of capsaicin treatment (white circles), training induced a progressive increase in flexion duration that reduced net shock exposure. Application of capsaicin (green circles) impaired learning. (E) Past exposure to controllable stimulation blocked the development of capsaicin-induced EMR. Spinally transected rats had received 30 min of instrumental training (Master, green), or nothing (Unshk, red), before the application of capsaicin to the same limb. Subjects were then tested by applying mechanical stimuli (von Frey) to the plantar surface of the injected and uninjected paw. Capsaicin treatment induced EMR in naïve (unshocked) subjects. Previous experience with controllable stimulation (Master) attenuated this effect. (F) Previous training with controllable stimulation blocked the capsaicin-induced learning impairment. Spinally transected rats were given 30 min of instrumental training (Master, green), or nothing (Unshocked, red), before the application of capsaicin to the same limb. Subjects were then tested by applying response-contingent shock to the contralateral leg. Capsaicin treatment impaired learning in the naïve (Unshocked) subjects. Past experience with controllable stimulation eliminated this learning impairment. EMR, enhanced mechanical reactivity. 
impairment in a dose-dependent manner. ${ }^{69}$ Surprisingly, administration of bicuculline also blocked the induction of the learning.

Other data, however, appeared to challenge the hypothesized link between the learning impairment and neural inhibition. We had previously shown that exposure to a long/intense continuous shock induces an inhibition of nociceptive reactivity (antinociception) in spinally transected rats. ${ }^{65,70}$ If exposure to VIS impairs learning because it inhibits nociceptive processing, it, too, should attenuate reactivity to noxious stimuli. Contrary to our hypothesis, VIS did not induce antinociception. ${ }^{41}$ Instead, it enhanced reactivity to mechanical stimulation applied to the plantar surface of the paw (Fig. 2C) ${ }^{46}$ As discussed above, EMR is associated with the sensitization of nociceptive circuits and the development of chronic pain. ${ }^{7,10}$ These observations suggested that exposure to VIS might impair learning because it induces a state akin to central sensitization, a lasting NMDAR-dependent overexcitation within the dorsal horn. ${ }^{46}$

In the hippocampal literature, it has been shown that diffusely saturating NMDAR-mediated plasticity blocks the development of LTP in selective pathways. $^{71}$ Likewise, saturating NMDARmediated plasticity within the spinal cord could impair learning that requires a selective modification within a particular stimulusresponse (S-R) pathway. This alternative account suggests that pretreatment with an NMDAR antagonist should block the induction of the learning impairment. To test this idea, we administered MK-801 i.t. before spinally transected rats received VIS to the tail. ${ }^{46}$ The next day, after the drug had cleared the system, rats were tested with controllable shock applied to one hindlimb. We found that MK-801 blocks the induction of the learning impairment in a dose-dependent manner.

If VIS impairs learning because it induces a central sensitizationlike state, then treatments that induce this state should impair instrumental learning. Supporting this, we have shown that peripheral application of an irritant (capsaicin, formalin, or cFA) impairs instrumental learning (Fig. 2D). ${ }^{45-47}$ Moreover, while the EMR and peripheral effects elicited by a moderate concentration of capsaicin fade within hours, the inhibition of learning lasts at least $24 \mathrm{~h}$, an outcome that parallels the long-term effect of VIS. ${ }^{41,45}$

The realization that the learning impairment is tied to nociceptive sensitization suggested a wealth of new ideas regarding the underlying neurochemical systems, concepts that built upon decades of work detailing the signal pathways that contribute to central sensitization. ${ }^{9}$ These links also highlighted the potential role of nonneuronal cells, which contribute to the gliopapthy that promotes nociceptive sensitization. ${ }^{19,72,73}$ Of particular interest was the cytokine, TNF, which has been linked to the metaplasticity and trafficking of $\alpha$-amino-3-hydroxy-5-methyl-4-isoxazolepropionic acid (AMPA) receptors (AMPARs) to the active zone of the synapse. ${ }^{74-77}$ If the learning impairment observed after VIS depends upon the release of TNF, then i.t. application of TNF should substitute for shock treatment and inhibit learning. Huie and colleagues showed that this was true. ${ }^{78}$ Further, like VIS, pretreatment with TNF induces a lasting $(>24 \mathrm{~h})$ learning impairment. Conversely, pretreatment with a soluble TNF receptor (sTNF), that sequesters free TNF, blocks both the induction and the expression of the learning impairment induced by VIS. $^{78}$

\section{Relational learning counters nociceptive sensitization}

An important implication of our work is that the impact of a noxious stimulus depends upon whether it is presented in a predictable/controllable or unpredictable/uncontrollable fashion. Rats that have received controllable noxious stimulation do not exhibit
EMR. ${ }^{45}$ Likewise, rats given an extended exposure to regular (temporally predictable) shocks are hypo-, rather than hyper-, reactive to mechanical stimulation. ${ }^{56}$ We have also shown that exposure to controllable/predictable stimulation has a lasting protective/restorative effect that counters the development of central sensitization. ${ }^{45,52,55,56,79,80}$ For example, Michelle Hook showed that training with controllable stimulation blocks the EMR, and learning impairment, induced by peripheral treatment with capsaicin (Fig. 2E,F) ${ }^{45}$ implying that relational learning attenuates the development of nociceptive sensitization (Fig. 2B).

We have also tested whether instrumental training can have a restorative effect that re-establishes the capacity to learn. To explore this issue, we needed a way to temporarily turn off the expression of the deficit. This was achieved by administering the opioid antagonist, naltrexone. ${ }^{67}$ As noted above, naltrexone blocks the expression, but not the induction, of the learning impairment. Using this pharmacological approach, we gave capsaicin-treated rats $30 \mathrm{~min}$ of instrumental training. When tested the next day (after the naltrexone had cleared the system), we found that rats that had received capsaicin alone exhibited a learning impairment. ${ }^{45} \mathrm{Im}$ portantly, this effect was eliminated by an intervening period of instrumental training.

Other studies have linked the protective/restorative effect of controllable stimulation to the expression of BDNF. Supporting this, pretreatment with i.t. BDNF can prevent, and reverse, the induction of the learning impairment induced by VIS. ${ }^{60}$ Conversely, the beneficial effect of instrumental training is blocked by i.t. application of the BDNF sequestering antibody, TrkB-IgG.

Parallel studies have been conducted examining the influence of temporal predictability. Baumbauer and colleagues showed that an extended exposure to shocks (720-900) presented at a regular (2sec) interval induces a lasting protective effect that counters the learning impairment and EMR induced by capsaicin. ${ }^{55,56}$ The induction of this lasting (24-h) effect has been shown to depend upon a form of NMDAR-mediated plasticity and protein expression. The expression of the protective effect has been linked to BDNF. ${ }^{52}$ Therapeutically, these observations are of interest because temporally regular stimulation could have a restorative effect in the absence of drug treatment or the capacity to perform a motor response.

\section{Spinal Injury Sets the Stage for Nociceptive Sensitization}

\section{Descending serotonergic fibers quell nociceptive sensitization}

Evidence suggests that brain-dependent processes normally quell the development of nociceptive sensitization through descending serotonergic fibers. ${ }^{21,81-85}$ Given this, it is not surprising that intermittent nociceptive stimulation has little effect on spinal plasticity if it is given before spinal transection. ${ }^{86}$ This brain-dependent protective effect has been related to serotonergic neuromodulation tied to fibers that descend through the dorsolateral funiculus (DLF). ${ }^{87}$ To explore the role of these fibers, rats received bilateral lesions of the DLF. The next day, rats were given VIS. The spinal cord was then transected and animals were tested in our instrumental learning paradigm $24 \mathrm{~h}$ later. As expected, VIS given before spinal injury had no effect on learning. In contrast, VIS induced a robust learning impairment in rats that had the DLF bilaterally lesioned. ${ }^{86}$

If the key descending fibers are serotonergic, i.t. application of a serotonergic agonist should have a protective effect and block the development of the learning impairment in spinally transected rats. We explored this possibility by administering $5 \mathrm{HT}$, or a selective 
agonist, before VIS in spinally transected rats. ${ }^{86}$ When tested the next day, we found pre-treatment with $5 \mathrm{HT}$, or a $5 \mathrm{HT}_{1 \mathrm{~A} / 7}$ agonist (8hydroxy-2-dipropylaminotetralin hydrobromide [8-OH-DPAT], i.t.), blocked the induction of the learning impairment. Because activation of the $5 \mathrm{HT}_{2}$ receptor has been shown to promote locomotor behavior, ${ }^{88,89}$ we also tested DIO $\left(5 \mathrm{HT}_{2}\right)$ and quipazine $\left(5 \mathrm{HT}_{2 / 3}\right)$. Neither blocked the induction of the learning impairment. ${ }^{86}$

Finally, we asked whether i.t. application of a $5 \mathrm{HT}_{1 \mathrm{~A}}$ antagonist (WAY 100635) blocks the brain-dependent protection of spinal circuits in uninjured rats. ${ }^{86}$ Intact rats received WAY 100635 , or its vehicle, before VIS and underwent a spinal transection $2 \mathrm{~h}$ later. As expected, vehicle-treated rats that were given VIS before a spinal transection showed no evidence of a learning impairment when tested with controllable stimulation. Uninjured rats that received the 5HT $1 \mathrm{~A}$ antagonist before VIS exhibited impaired learning. Taken together, these observations show that descending 5HT fibers within the DLF normally exert a protective effect. Likewise, descending 5HT fibers have been shown to inhibit the development of spinally mediated LTP and cellular indices of nociceptive sensitization. ${ }^{21,81-85}$

\section{Injury transforms GABAergic regulation of nociceptive circuits}

We have built a strong case linking the learning impairment to the induction of a central sensitization-like state. We have shown that VIS induces EMR and that a variety of treatments known to produce central sensitization (e.g., peripheral application of capsaicin) impair learning. ${ }^{45,46}$ Building upon these similarities, we have demonstrated that pharmacological manipulations that attenuate central sensitization also block the VIS-induced learning impairment. We have further shown that spinally mediated relational learning can counter the learning impairment and EMR induced by capsaicin.

Our findings concur with the larger literature demonstrating that brain-dependent processes regulate nociceptive circuits within the spinal cord and act to quell sensitization. ${ }^{16,85}$ From this perspective, SCI (and, in particular, damage to the descending serotonergic processes) prepares the stage for the development of central sensitization, by releasing the system from a source of inhibition. The corollary to this is that spinally mediated nociceptive sensitization may, under normal circumstances, only arise post-SCI-that SCI is an essential premorbid condition.

How does the disruption of descending serotonergic fibers alter spinal function, allowing nociceptive stimulation to induce a learning impairment and EMR? One possibility is that SCI transforms the cellular environment within the spinal cord, impacting circuit function and setting the stage for nociceptive sensitization. Recently, Yung-Jen Huang showed that these effects may be related to an alteration in GABA function. ${ }^{96}$ Recall that we have shown that administration of a GABA A antagonist (bicuculline) attenuates the learning deficit. ${ }^{69}$ At the time, this study was motivated by the idea that VIS impaired instrumental learning because it engaged GABAergic interneurons within the spinal cord that inhibited learning. Consistent with this, we showed that bicuculline administered before testing blocked the expression of the learning impairment. ${ }^{69}$ What seemed surprising is that the administration of bicuculline before VIS blocked the learning impairment when rats were tested $24 \mathrm{~h}$ later, after the drug had cleared the system. If GABA is inhibitory, why would removing a source of inhibition during VIS block the development of the learning impairment? These observations seem even more paradoxical when they are coupled with our subsequent data, linking the learning deficit to the development of central sensitization. Indeed, if the latter claim has merit, our find- ings would appear to suggest that administration of bicuculline would block the induction and expression of central sensitization.

At a conceptual level, the idea that blocking GABAergic inhibition should attenuate overexcitation and central sensitization made no sense. After all, administration of a GABA antagonist typically enhances neural excitability and GABA agonists (e.g., muscimol) are routinely used to locally inhibit neural function. At an empirical level, previous work has shown that pre-treatment with bicuculline generally enhances reactivity to nociceptive stimulation and neural excitability within the spinal cord. ${ }^{90-94}$ We recently replicated this observation, demonstrating that in uninjured rats i.t. bicuculline per se enhances reactivity to tactile stimulation and, if anything, promotes the development of central sensitization. ${ }^{95,96}$

The problem here may lie with the assumption that GABA has an inhibitory effect post-SCI. GABA function is regulated by two cotransporters, $\mathrm{K}^{+}-\mathrm{Cl}^{-}$cotransporter $2(\mathrm{KCC} 2)$ and $\mathrm{Na}^{+}-\mathrm{K}^{+}-\mathrm{Cl}^{-}$ cotransporter 1 (NKCC1). ${ }^{97}$ The inward flow of $\mathrm{Cl}^{-}$is regulated by NKCC1 whereas the outward flow is controlled by KCC2 (Fig. 3A). In the adult nervous system, these cotransporters work together to maintain a low intracellular concentration of $\mathrm{Cl}^{-}$. Under these conditions, engaging the iontropic GABA-A receptor allows $\mathrm{Cl}^{-}$to flow into the cell, causing the hyperpolarization that underlies its inhibitory effect on neural transmission. Interestingly, early in development, the membrane-bound levels of KCC2 are much lower and, as a result, the outward flow of $\mathrm{Cl}^{-}$is reduced. ${ }^{98,99}$ This leads to a rise in intracellular $\mathrm{Cl}^{-}$concentrations. The result is a flip in GABA-A receptor function; when it is engaged, it allows $\mathrm{Cl}^{-}$to exit the cell, which has a depolarizing effect.

Recent studies have shown that spinal injury can cause a downregulation in membrane-bound $\mathrm{KCC} 2$, producing an increase in neural excitability that has been linked to the development of chronic pain. ${ }^{97,100-103}$ However, the focus of these studies was on the development of spontaneous pain weeks post-injury. Our question differed in two regards. First, we were concerned with the acute effects of spinal injury and how this transforms the cellular milieu (to affect plasticity, recovery, and pain). Second, we sought to elucidate how an acute change in GABA function affects the development of nociceptive sensitization. To address these issues, we examined whether SCI induces a rapid shift in membranebound KCC2. ${ }^{96,104}$ Adult rats received a spinal transection at T2 or underwent a sham surgery. A day later, the lumbosacral tissue was collected and separated into cytoplasmic and membrane fractions. KCC2 levels were then assayed using western blotting. We found that the ratio of membrane to cytoplasmic $\mathrm{KCC} 2$ was significantly lower $24 \mathrm{~h}$ after a spinal transection.

Our claim is that a reduction in membrane-bound $\mathrm{KCC} 2$ will reduce GABAergic inhibition. We tested this idea by comparing the effect of a GABA agonist (muscimol) on mechanical reactivity in sham-operated and spinally transected rats. As expected, i.t. muscimol inhibited mechanical reactivity and the development of capsaicin-induced nociceptive sensitization in sham-operated rats. ${ }^{96,105}$ Spinal transection eliminated this inhibitory effect.

Next, we examined the impact of the GABA-A antagonist, bicuculline, on capsaicin-induced EMR in spinally transected rats. In the absence of injury, bicuculline induces a state akin to central sensitization. ${ }^{93}$ Yet, after spinal injury, we are led to a very different prediction. As noted above, we have shown that bicuculline blocks the induction and expression of the learning impairment. We have also shown that treatments that induce nociceptive sensitization impair learning. If these phenomena are related, bicuculline should attenuate the development of capsaicin-induced EMR in spinally injured rats. We found that bicuculline has this effect. ${ }^{96,105}$ 
A Injury Increases Intracellular $\mathrm{Cl}^{-}$Concentration

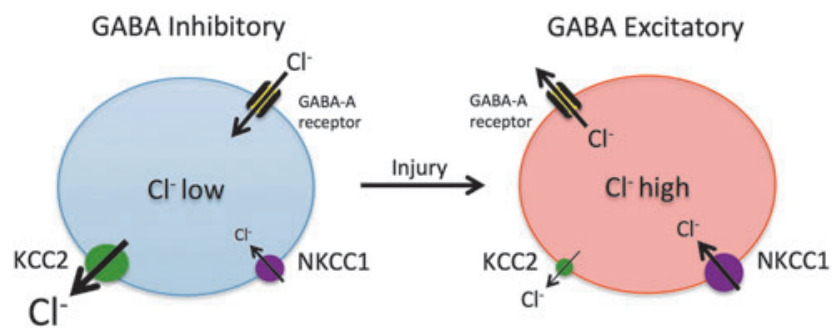

Block KCC2 with DIOA $\rightarrow$

$\leftarrow$ Block NKCC1 with bumetanide

C Spinal Injury Transforms the Impact of Drug Treatments

\begin{tabular}{|c|c|c|}
\hline Treatment & Uninjured & Injured \\
\hline Capsaicin alone & $\uparrow E M R, \uparrow E R K$ & 个 EMR, 个个 ERK \\
\hline Bicucculline $\rightarrow$ Capsaicin & 个 EMR, -- ERK & $\downarrow \downarrow E M R, \downarrow$ ERK \\
\hline Muscimol $\rightarrow$ Capsaicin & $\downarrow \downarrow E M R$ & --- EMR \\
\hline BDNF $\rightarrow$ Capsaicin & 个 EMR, 个ERK & $\downarrow \downarrow E M R, \downarrow$ ERK \\
\hline
\end{tabular}

B Intracellular $\mathrm{Cl}^{-}$Alters GABA Feedback

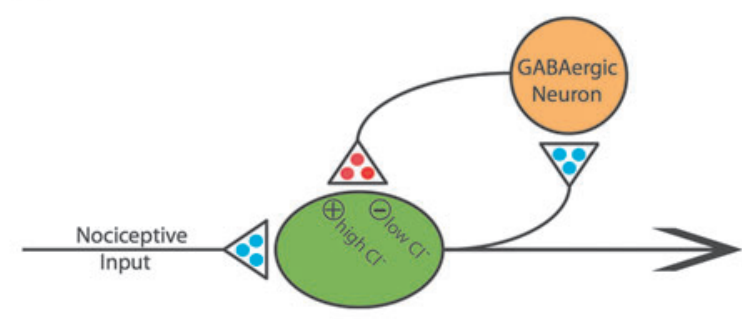

D Evaluating Drug Effect in a Choice Task Condition

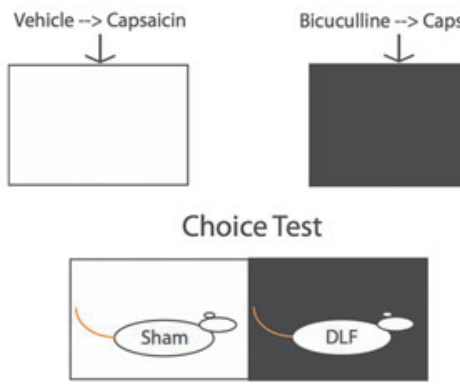

FIG. 3. GABA function is transformed by spinal injury. (A) In adult uninjured animals (left), the combined action of the cotransporters KCC2 and NKCC1 maintain a low intracellular concentration of $\mathrm{Cl}^{-}$. Engaging the GABA-A receptor allows $\mathrm{Cl}^{-}$to flow into the cell, causing the hyperpolarization that underlies its inhibitory effect. Spinal injury (right) lowers membrane-bound KCC2, which reduces the outward flow of $\mathrm{Cl}^{-}$and increases intracellular $\mathrm{Cl}^{-}$concentrations. Under these conditions, engaging the $\mathrm{GABA}-\mathrm{A}$ receptor can allow $\mathrm{Cl}^{-}$to exit the cell, which would have a depolarizing effect. Intracellular $\mathrm{Cl}^{-}$concentrations can be experimentally manipulated in adult animals using drugs that disrupt KCC2 (DIOA) and NKCC1 (bumetanide) function. In intact animals, DIOA retards the outward flow of $\mathrm{Cl}^{-}$through the $\mathrm{KCC} 2$ channel, which increases intracellular $\mathrm{Cl}^{-}$concentrations (bottom). In spinally transected animals, bumetanide lowers the flow of $\mathrm{Cl}^{-}$into the cell, reducing the intracellular $\mathrm{Cl}^{-}$concentration. (B) In uninjured animals, GABAergic neurons within the spinal cord are engaged by afferent nociceptive input and may provide a form of negative feedback, which would help prevent overexcitation. After spinal injury, the reduction in membrane-bound KCC 2 would transform how GABA release affects nociceptive circuits, causing it to have a depolarizing [excitatory (+)] effect that could contribute to the development of nociceptive sensitization. Excitatory (glutamatergic) transmitters are indicated in blue and inhibitory (GABAergic) transmitters are colored red. (C) Summary of how alternative treatments affect capsaicin-induced EMR and ERK activation in uninjured and spinally injured rats. Stronger effects are indicated with a double arrow. A dashed line indicates that a drug treatment has no effect. (D) The task used to examine how alterations in spinal GABA function affect pain signals relayed to the brain. Behavioral data indicated that lesioning the DLF transformed how i.t. bicuculline affects capsaicin-induced EMR, causing it to have an anti-allodynic-like effect. If this modification also alters pain signaling to the brain, i.t. bicuculline should attenuate capsaicin-induced pain and reduce its capacity to support a conditioned aversion. To evaluate this possibility, DLF-lesioned and sham-operated rats were given bicuculline or its vehicle i.t. before capsaicin treatment and being placed in one of two distinctive contexts. Each subject received both types of conditioning trials, which were spaced across days. Both treatment order and which environment was paired with bicuculline were counterbalanced across subjects. Rats were then given a choice between the two conditioning chambers. As predicted, DLF-lesioned rats preferred the environment where they had experienced i.t. bicuculline before capsaicin treatment. If anything, sham-operated rats exhibited the opposite preference. BDNF, brain-derived neurotrophic factor; DIOA, dihydro-indenyl-oxy alkanoic acid; DLF, dorsolateral funiculus; EMR, enhanced mechanical reactivity; ERK, extracellular signal-regulated kinases; GABA, gamma-aminobutyric acid; GABA-A, GABA type A; i.t., intrathecal; $\mathrm{KCC} 2, \mathrm{~K}^{+}-\mathrm{Cl}^{-}$cotransporter 2 ; $\mathrm{NKCC} 1, \mathrm{Na}^{+}-\mathrm{K}^{+}-\mathrm{Cl}^{-}$cotransporter 1 .

As discussed above, nociceptive sensitization within the spinal dorsal horn is associated with increased expression of the transcription factor, $c$-fos, and the phosphorylation of ERK. To reinforce our behavioral data, we examined the impact of bicuculline treatment on these cellular markers. ${ }^{96}$ Our assays revealed two interesting findings. First, the magnitude of $c$-fos expression and ERK activation (the ratio of pERK to ERK) in response to capsaicin treatment was generally greater after spinal injury. Second, the impact of i.t. bicuculline on these cellular indices of central sensitization paralleled our behavioral data; i.t. bicuculline attenuated $c$ fos and pERK expression in spinally transected, but not intact, rats.

We have reinforced our findings using drug manipulations that target the KCC2 and NKCC1 cotransporters. ${ }^{96,104}$ If SCI transforms how bicuculline acts because it reduces membrane-bound $\mathrm{KCC} 2$, then blocking KCC2 function in uninjured rats with dihydroindenyl-oxy alkanoic acid (DIOA) should produce the same effect. ${ }^{101}$ As predicted, pretreating intact rats with DIOA (i.t.) eliminated bicuculline-induced EMR and reversed its effect on capsaicin-induced EMR, yielding a pattern identical to that observed post-SCI. ${ }^{96}$ This line of thought also suggests that we could compensate for an injury-induced downregulation of membrane-bound KCC2 by administering a drug, bumetanide, that disrupts NKCC1 function. Because bumetanide reduces the inward flow of $\mathrm{Cl}^{-}$, it lowers intracellular $\mathrm{Cl}^{-}$concentrations and acts to re-establish GABAergic inhibition. ${ }^{102}$ This suggests that bumetanide should reverse how bicuculline affects nociceptive reactivity. As predicted, 
bicuculline enhanced mechanical reactivity in injured rats pretreated with bumetanide (i.t.) and failed to block capsaicin-induced EMR, ${ }^{96}$ a pattern of results that parallels the effect of bicuculline in uninjured rats. By helping to restore GABAergic inhibition post-injury, bumetanide treatment could have therapeutic value.

It is easy to see how a loss of GABAergic inhibition after spinal injury would eliminate bicuculline-induced EMR — we just need to assume that GABA release provides a source of tonic inhibition. An injury-induced downregulation in $\mathrm{KCC} 2$ would attenuate this inhibitory effect and reduce the bicuculline-induced EMR. What is more difficult to explain is why bicuculline blocks capsaicininduced EMR after spinal injury. The implication is that GABA is playing an essential role in the induction of nociceptive sensitization. As discussed elsewhere,${ }^{96}$ there are a variety of potential accounts of this effect. One simple possibility is illustrated in Figure 3B, which assumes that a subset of GABAergic interneurons provides a form of negative feedback in the uninjured system. Here, nociceptive input would engage GABAergic neurons that project back to regulate afferent input, reducing transmitter release and/or depolarization in fibers that drive motor output and nociceptive signaling. In the uninjured system, this negative feedback could function to regulate the development of nociceptive sensitization and serve to prevent overexcitation. Post-injury, the downregulation of $\mathrm{KCC} 2$ would eliminate this brake on nociceptive transmission and, to the extent GABA now has a depolarizing effect, contribute to the development of nociceptive sensitization. The implication is that, after spinal injury, GABA could function as a kind of gas pedal rather than a brake. From this perspective, bicuculline blocks the induction of central sensitization, and the resultant learning impairment, because it disables this gas pedal.

Our results may also be relevant to studies examining the effect of injury during early development. Young and colleagues showed that rats given a peripheral injury (removal of a small flap of tissue from the plantar surface of one hindpaw) at postnatal day 2 exhibit EMR when tested at 12 weeks of age. ${ }^{106}$ This effect survived a spinal transection, implying that the early injury produced a lasting change in spinal function. Further, the EMR observed in injured rats was accompanied by an impairment in spinal learning. ${ }^{106,107}$ Early injury may have these effects because the GABAergic system is still maturing, with lower levels of membrane-bound $\mathrm{KCC} 2$ and reduced inhibition. The resultant state would be analogous to that observed post-SCI, enabling noxious input to have a more-robust and longer-lasting effect.

\section{Injuries limited to the dorsolateral funiculus transform GABA and nociceptive signaling}

We argued earlier that the brain regulates the development of spinally mediated nociceptive sensitization through serotonergic fibers within the DLF. The loss of this input could contribute to the transformation in GABA function. To explore this possibility, we assessed the impact of i.t. administration of a $5 \mathrm{HT}_{1 \mathrm{~A}}$ agonist (8OH-DPAT, i.t.) on $\mathrm{KCC} 2$ in spinally transected rats. We found that the drug increased membrane-bound KCC2 levels and transformed how bicuculline affects nociceptive reactivity, yielding a pattern of results indicative of increased GABAergic inhibition. ${ }^{108}$ Conversely, i.t. administration of a $5 \mathrm{HT}_{1 \mathrm{~A}}$ antagonist (WAY 100635) flipped how bicuculline affected nociceptive reactivity in uninjured rats, generating a pattern of results identical to those obtained after spinal transection. Finally, lesions limited to the DLF are sufficient to downregulate membrane-bound $\mathrm{KCC} 2$ and switch how i.t. bicuculline affects nociceptive sensitization. ${ }^{109}$
The fact that GABA function can be transformed by DLF lesions has allowed us to address an issue that was otherwise impossible to tackle. Our claim is that an injury-induced reduction in spinal $\mathrm{KCC} 2$ promotes the development of nociceptive sensitization because GABA has a depolarizing effect. If this is true, administration of a GABA-A antagonist should attenuate both capsaicin-induced EMR and nociceptive transmission to the brain. Under these conditions, bicuculline should reduce, rather than enhance, pain. Because DLF lesions appear to downregulate $\mathrm{KCC} 2$, while preserving ascending sensory fibers, we can use this manipulation to investigate how an alteration in spinal levels of membrane-bound KCC2 affects braindependent measures of pain signaling. We addressed this issue using a place conditioning paradigm. ${ }^{109,110}$ Rats received bilateral lesions of the DLF or a sham surgery. ${ }^{110}$ Over the next 2 days, they were given bicuculline, or its vehicle, before capsaicin was applied to one hindpaw and being placed in a distinct chamber (black or white; Fig. 3D). In this type of conditioning task, experimental treatments that enhance the affective impact of a nociceptive stimulus promote the development of a conditioned aversion to cues associated with the experience. Conversely, treatments that reduce pain attenuate aversive conditioning. To examine how bicuculline treatment affected conditioning, we then allowed the rats to choose between the black and white chambers. As expected, sham-operated rats showed an aversion to the chamber that was paired with bicuculline, implying that the drug treatment had enhanced pain. Rats that had received lesions limited to the DLF showed the opposite pattern of results; they preferred the bicuculline paired chamber, implying that the drug treatment had attenuated pain. ${ }^{109}$

\section{Injury transforms how brain-derived neurotrophic factor affects nociceptive processing}

We showed above that relational learning has a protective/restorative effect that counters the learning impairment and EMR induced by capsaicin. ${ }^{45,55,56}$ The former effect was linked to an upregulation of BDNF. Supporting this, we showed that exogenous BDNF blocks the induction of the learning impairment in spinally transected rats. ${ }^{60}$ If the learning impairment and EMR are linked to a common process (central sensitization), then exogenous BDNF should also block capsaicin-induced EMR. Recently, we reported this pattern of results in spinally transected rats. ${ }^{111}$ However, others have argued that BDNF fosters the development of central sensitization and linked this effect to a downregulation in membranebound KCC2. ${ }^{112-115}$ These opposing findings suggest that spinal injury transforms how BDNF affects spinal function. However, because the studies differed on many dimensions (e.g., dose, time of testing), no strong conclusions could be drawn. We addressed this issue by comparing the impact of i.t. BDNF in spinally transected and sham-operated rats. BDNF downregulated membranebound $\mathrm{KCC} 2$ in intact (sham-operated) rats and had the opposite effect post-SCI. ${ }^{116}$ Likewise, BDNF enhanced mechanical reactivity and capsaicin-induced EMR in sham-operated rats, but attenuated capsaicin-induced EMR after spinal injury. In simple terms, in uninjured rats, i.t. BDNF produces an effect similar to that produced by spinal injury-generating a cellular environment conducive to the development of nociceptive sensitization. In contrast, after spinal injury, BDNF appears to have a homeostaticlike effect that helps to restore GABAergic inhibition. Under these conditions, BDNF counters the development of nociceptive sensitization. These observations suggest that the usefulness of BDNF as a therapeutic will depend upon injury severity. BDNF may generally promote spinal functions soon after a severe contusion injury or 
when descending fibers are cut. In contrast, when damage to descending fibers is minimal, or has recovered, membrane-bound $\mathrm{KCC} 2$ and GABAergic inhibition will return to normal. As it does, increased BDNF expression could promote nociceptive sensitization and the development of chronic pain.

We have shown that spinal injury, and the loss of descending $5 \mathrm{HT}$ fibers, brings about a change in membrane-bound $\mathrm{KCC} 2$ that alters GABAergic function (Fig. 3C). SCI also transforms the action of BDNF; in intact animals, BDNF expression promotes the development of nociceptive sensitization, whereas after spinal transection, BDNF counters overexcitation and promotes spinally mediated learning. ${ }^{59,60}$ Here, too, the transformation in function was related to alterations in membrane-bound $\mathrm{KCC} 2$. These results are consistent with other findings demonstrating that SCI-induced spasticity is related to a BDNF-dependent reduction in membranebound KCC2. ${ }^{101}$ These neurochemical processes also appear to contribute to the allodynia observed in diabetic rats. ${ }^{117}$ It has been suggested that the opposing effects of BDNF on $\mathrm{KCC} 2$ may be related to phospholipase $\mathrm{C}-\gamma$ (PLC- $\gamma$ ), an effector of TrkB signaling; BDNF upregulates KCC2 in the presence of PLC- $\gamma$, but downregulates KCC2 when PLC- $\gamma$ is absent. ${ }^{18,119}$ Further work is needed to elucidate the essential signal pathways and how they transform BDNF function.

\section{Implications for Recovery After a Contusion Injury}

Our work suggests that noxious stimulation has an exaggerated effect on nociceptive circuits post-SCI that drives overexcitation, fosters the development of nociceptive sensitization, and interferes with spinally mediated learning. Because the consequences of uncontrollable/unpredictable stimulation are long-lasting, and because nociceptive sensitization can lead to chronic pain, we have suggested that it represents an example of maladaptive plasticity. ${ }^{80}$ Conversely, learning about temporal and R-O relations appears adaptive in nature because it enables selective response modifications that reduce net exposure to noxious stimulation and has a lasting protective/restorative effect that counters the development of nociceptive sensitization (Fig. 2B). ${ }^{120}$

We have detailed how these processes work using a transection paradigm, demonstrating that learning can occur without input from the brain. ${ }^{27,55,120,121}$ This work reinforces the idea that physical rehabilitation can benefit recovery after severe injury by engaging spinal circuits caudal to injury. ${ }^{39,122}$ Our work also reminds us that noxious stimulation can impact neural function in the absence of pain reports and that its adverse effect can be minimized by presenting stimuli in a predictable/controllable manner. In the sections that follow, we show that these findings are also relevant to recovery after a contusion injury.

\section{Uncontrollable nociceptive stimulation impairs recovery}

Our hypothesis was that unregulated nociceptive input caudal to a contusion injury could induce a state of neural overexcitation that enhances cell loss, impairs recovery, and promotes chronic pain. To explore these issues, rats received a lower thoracic contusion injury. ${ }^{123} \mathrm{~A}$ day post-injury, we applied VIS to the tail. Locomotor function, and other indices of behavioral recovery, were then assessed over the next 6 weeks. ${ }^{123}$ Our moderate injury produced a disruption in motor function that waned over the subsequent 2 weeks, with some recovery of weight-supported stepping (Fig. 4A). Nociceptive stimulation caused an acute decline in performance and impaired behavioral recovery. We have recently shown that peripheral application of capsaicin to one hindpaw a day postinjury has a similar effect. ${ }^{124}$

To explore whether the effect of noxious stimulation varies over time, separate groups of rats received VIS 1,4 , or 14 days after a contusion injury. ${ }^{123} \mathrm{We}$ found that nociceptive stimulation has a greater effect when given 1-4 days post-injury. This suggests that stimulation affects long-term recovery because it impacts processes (e.g., secondary injury; Fig. 4B) that are triggered by the primary physical injury and that unfold over hours to days post-injury.

A basic implication of our work is that the impact of noxious stimulation is regulated by relational learning; that uncontrollable stimulation engages a form of maladaptive plasticity whereas the same stimulus given in a controllable manner has no adverse effect. To explore whether learning also regulates the effect of noxious stimulation on recovery, one group (Master) of contused rats was given controllable leg shock. Rats in a second group (Yoked) were experimentally coupled to the master rats and received shock at the same time and for the same duration. We found that only uncontrollable stimulation adversely affects locomotor recovery. ${ }^{123}$

To evaluate the effect of uncontrollable nociceptive stimulation on nociceptive processing, ${ }^{125}$ we assessed reactivity to mechanical stimulation applied to the hindpaw. As reported by others, enhanced mechanical reactivity emerged 2-4 weeks post-injury alone (Fig. 4C), an effect that has been related to the development of allodynia and chronic pain. ${ }^{7,126}$ Rats that had received nociceptive stimulation a day after a contusion injury exhibited an EMR that emerged sooner (at 7 days) and that was significantly stronger 2-4 weeks post-injury. ${ }^{125}$

Other indices of recovery reinforce the idea that nociceptive input soon after injury has a damaging effect. Both the recovery of weight and bladder function were impaired in contused rats that received shock. ${ }^{123}$ Nociceptive stimulation also increased the incidence of spasticity and reduced the amount of tissue (white and gray matter) spared at the site of injury. An increase in tissue loss again implies that noxious input affects the development of secondary injury.

\section{Noxious stimulation reduces brain-derived neurotrophic factor and increases tumor necrosis factor}

In spinally transected rats, we have related the beneficial effect of controllable/predictable stimulation to an upregulation of BDNF expression within the lumbosacral region and shown that uncontrollable stimulation downregulates BDNF messenger RNA (mRNA) levels. In contused rats, BDNF mRNA expression was downregulated $48 \mathrm{~h}$ post-injury and protein expression was lowered from 1 to 8 days. ${ }^{127}$ More important, nociceptive stimulation further reduces BDNF mRNA expression at $24 \mathrm{~h}$ and BDNF protein from 1 to 7 days after shock treatment. BDNF interacts with the TrkB receptor and it, too, was downregulated by injury per se. Nociceptive stimulation further reduced TrkB mRNA expression within the dorsal horn at $24 \mathrm{~h}$ and protein 1-7 days after shock treatment. Signal pathways engaged by BDNF (e,g., CaMKII and ERK2) were also downregulated within the dorsal horn by nociceptive stimulation. $^{127}$

We have related the learning impairment to an upregulation of the cytokine, $\mathrm{TNF}^{78} \mathrm{TNF}$ is of particular interest because it can foster neural excitation, the development of central sensitization and cell death, and is associated with the development of neuropathic pain in humans. ${ }^{74-77,128}$ Garraway and colleagues showed that nociceptive stimulation increases TNF mRNA from 1 to 7 days in contused rats. ${ }^{125}$ TNF protein expression was enhanced within $1 \mathrm{~h}$ of noxious 
A Nociceptive Stimulation Impairs Recovery

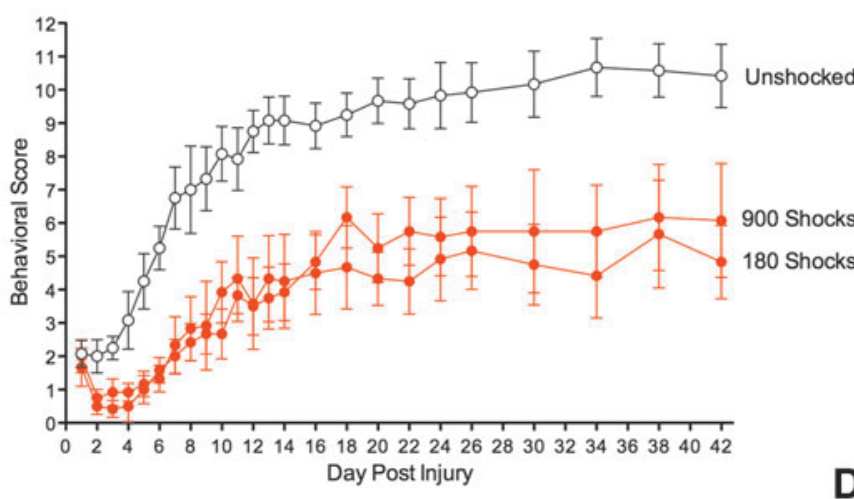

\section{Nociceptive Stimulation Enhances Allodynia}

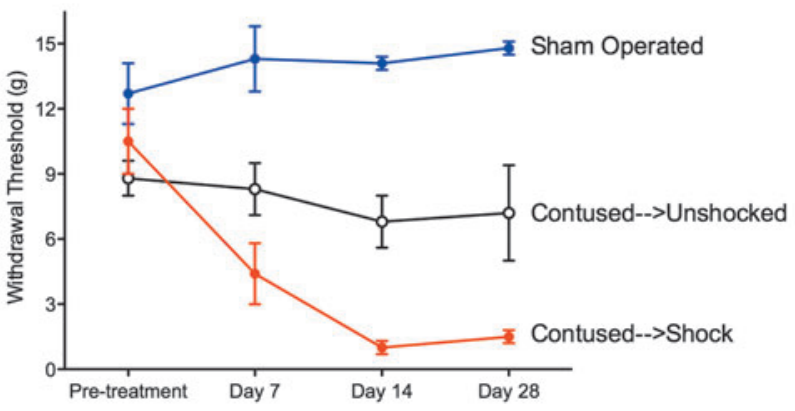

B Nociceptive Stimulation Increases $2^{\circ}$ Injury

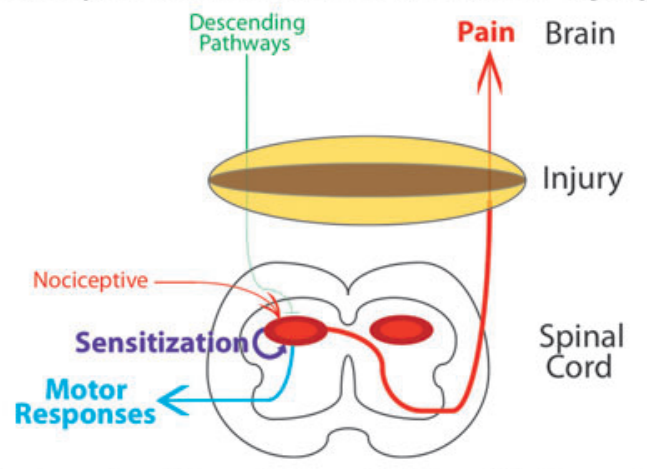

Nociceptive Stimulation $\rightarrow$ Blood in Samples

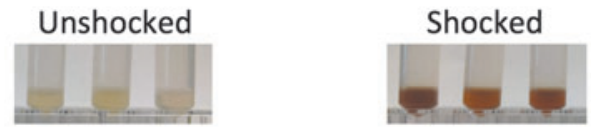

E Nociceptive Stimulation Increases Hemorrhage
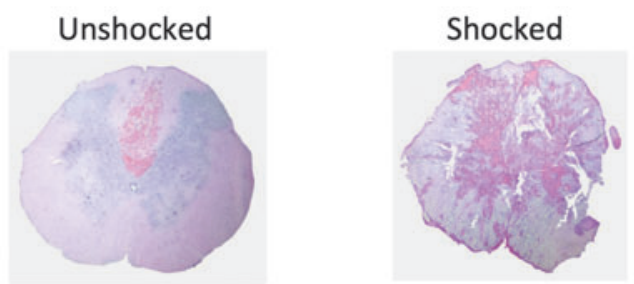

FIG. 4. Impact of nociceptive stimulation on recovery and secondary injury. (A) Nociceptive stimulation after a contusion injury impaired the recovery of locomotor function. VIS (180 or 900 shocks, red) was applied to contused rats a day after injury and locomotor function was monitored for 6 weeks using the BBB locomotor scale. ${ }^{159,160}$ Unshocked rats (white circles) exhibited some recovery of weight-supported stepping. Animals that had received VIS exhibited an acute decline in locomotor function and never recovered to the same level as the Unshocked controls. (B) After a contusion injury, nociceptive stimulation can sensitize nociceptive circuits caudal to injury and expand the region of secondary injury (yellow). (C) Relative to unshocked sham-operated rats (blue), unshocked contused rats (white circles) are more responsive to mechanical stimuli applied to the plantar surface of a hind paw 2-4 weeks post-injury. Rats that had received nociceptive stimulation (Shock, red) exhibited an EMR that emerged sooner (at 7 days) and was more robust at 2-4 weeks post-injury. (D) Nociceptive stimulation induces hemorrhage at the site of injury. One day after a contusion injury, rats received 6 min of VIS (Shocked) or nothing (Unshocked). Samples collected $3 \mathrm{~h}$ after shock treatment were tinted red. (E) Representative hematoxylin and eosin-stained sections from contused rats that had received nociceptive stimulation (Shocked), or nothing (Unshocked), $3 \mathrm{~h}$ earlier. Shock treatment increased the area of hemorrhage at the site of injury. BBB, Basso, Beattie, Bresnahan; EMR, enhanced mechanical reactivity; VIS, variable intermittent stimulation.

stimulation and remained elevated for $24 \mathrm{~h}$. Immunolabeling revealed that noxious stimulation induced heavy labeling for TNF in the superficial dorsal horn. Labeling was colocalized with a marker for microglia, but not neurons or astrocytes. ${ }^{125}$

TNF can engage either TNF receptor 1 (TNFR1) or receptor 2 (TNFR2). These two receptors differ in how they are activated by $\mathrm{TNF}$, distributed within the spinal cord, and their presumed function. ${ }^{129-133}$ TNFR 1 is activated by sTNF and has been linked to both proinflammatory effects and programmed cell death (apoptosis). TNFR2 is activated by membrane-bound TNF and has been linked to anti-inflammatory/protective processes after neural insult. Whereas TNFR1 is constitutively expressed within a number of cell types throughout the spinal cord, TNFR2 is largely restricted to hematopoietic and endothelial cells. ${ }^{129,130}$ Because exogenously applied sTNF can substitute for VIS and induce a lasting learning impairment, we have suggested that the adverse effect of nociceptive stimulation is tied to TNFR $1{ }^{78}$ Engaging this receptor could foster the development of maladaptive plasticity by increasing the expression of $\mathrm{Ca}^{++}$-permeable AMPARs. ${ }^{134}$ TNFR1 activation could also contribute to cell loss post-injury by promoting apoptosis through the activation of the caspase-8/caspase- 3 signaling pathway. ${ }^{135-137}$ To explore this possibility, Garraway and colleagues assessed caspase expression after a contusion injury. ${ }^{125}$ Caspase $8 \mathrm{mRNA}$ and protein expression were increased 7-28 days postinjury. However, soon after injury, only rats given VIS exhibited increased caspase- 8 expression. Likewise, injury per se increased the expression of the active forms of caspase-3 (12 and $17 \mathrm{kDa})$ and this effect was observed 7 days post-injury. Exposure to nociceptive stimulation a day after injury caused a rapid increase in caspase-3 expression that emerged within an hour and remained elevated for $24 \mathrm{~h}$. Immunolabeling revealed caspase- 3 expression in neurons and microglia, but not astrocytes, $24 \mathrm{~h}$ after nociceptive stimulation. ${ }^{125}$ These results suggest that nociceptive stimulation leads to increased cell loss at the site of injury because it induces apoptosis.

\section{Noxious stimulation induces cell death and hemorrhage}

Recent studies suggest that an alternative form of cell death, pyroptosis, may also contribute to cell loss after SCI. ${ }^{138-140}$ 
Pyroptosis is linked to the formation of an inflammasome that promotes activation of caspase- 1 and the release of interleukin- 1 beta (IL-1B) and interleukin-18 (IL-18). These processes upregulate the expression of TNF and lead to the formation of 1- to 2-nm plasma membrane pores that allow the entry of molecules (e.g., $\mathrm{Ca}^{++}$) that initiate cell lysis. This induces a form of positive feedback that can drive overexpression of TNF, promote cell death, and foster the development of central sensitization. ${ }^{19}$ To explore whether nociceptive stimulation induces pyroptosis at the site of contusion injury, Turtle and colleagues assessed caspase-1, IL-1ß, and IL-18 expression. Caspase-1 expression was increased 1,3 , and $24 \mathrm{~h}$ post-VIS. ${ }^{141}$ Likewise, nociceptive stimulation activated IL-1B and IL-18. A similar pattern was observed in contused rats treated with the irritant capsaicin applied to one hindpaw a day post-injury. Taken together, it appears that nociceptive stimulation promotes pyroptosis as well as apoptosis. We are currently exploring whether drug treatments (e.g., administration of Brilliant Blue G) that attenuate pyroptotic cell death reduce the adverse effect of noxious input post-SCI.

In the course of conducting these cellular assays, Joel Turtle made a surprising observation-that homogenized tissue samples from rats that had received nociceptive stimulation were tinted red (Fig. 4D). ${ }^{142}$ Naturally, he hypothesized that this was related to the infiltration of red blood cells. Supporting this, he showed that samples from animals that had received nociceptive stimulation exhibited greater absorbance at the wavelength $(420 \mathrm{~nm})$ associated with this cell type. A similar effect was observed in contused rats that were treated with the irritant, capsaicin. ${ }^{142}$ Protein assays for hemoglobin confirmed that rats that had received nociceptive stimulation had higher levels. To further explore these effects, Melissa Brumley examined tissue sections stained with hematoxylin and eosin. ${ }^{142}$ She found that nociceptive stimulation increases the area of hemorrhage in contused rats (Fig. 4E).

Tissue hemorrhage post-SCI reflects the combined effect of the primary injury (primary hemorrhage) and subsequent processes that expand the affected region (secondary hemorrhage). The latter effect involves progressive hemorrhagic necrosis (PHN), which is linked to a catastrophic failure of capillary structure. ${ }^{143}$ This effect has been tied to the expression of sulfonylurea receptor $1 /$ transient receptor potential melastatin 4 (Sur1-Trpm4) channels in endothelial cells, which triggers necrotic cell death and capillary fragmentation. Preliminary data indicate that nociceptive stimulation after a contusion injury increases Sur1-Trpm4 expression. ${ }^{144}$ This observation suggests that treatments that inhibit Sur1-Trpm4 activation (e.g., glibenclamide) could attenuate the adverse effect nociceptive stimulation has on recovery. Our findings are consistent with other work demonstrating that noxious input increases the permeability of the blood-spinal cord barrier in uninjured rats. ${ }^{145}$ This effect could interact with SCI to produce PHN.

\section{Lidocaine, but not morphine, blocks the effect of acute noxious input}

Many SCIs are accompanied by additional sources of tissue damage (polytrauma) that will provide a source of noxious input, which, we have shown, can impair recovery by enhancing cell loss at the site of injury. We hypothesized that the adverse effect of noxious input could be attenuated using an opiate analgesic. To test this hypothesis, we administered a dose of morphine that completely blocked nociceptive reactivity to a noxious thermal stimulus applied to the tail. ${ }^{146}$ Morphine treatment also blocked behavioral reactivity to intermittent shock in contused rats. Morphine did not, however, block the effect of shock on locomotor recovery. More alarming, morphine-treated rats that had received nociceptive stimulation exhibited a higher rate of mortality and this effect emerged days after drug treatment. Further work showed that morphine per se, when applied i.t. a day post-injury, adversely affects behavioral recovery, and increases tissue loss, in contused rats. These effects have been linked to IL-1ß expression and the activation of the kappa opioid receptor. ${ }^{147,148}$ We have also shown that morphine treatment does not attenuate shock-induced PHN or the expression of cytokines related to pyroptotic cell death. ${ }^{149}$

As an alternative treatment, we have examined the impact of the $\mathrm{Na}^{+}$channel blocker, lidocaine, administered i.t. $30 \mathrm{~min}$ before nociceptive stimulation. ${ }^{149}$ Like morphine, lidocaine completely blocked behavioral reactivity to shock stimulation in contused rats. Unlike morphine, lidocaine also blocked shock-induced hemorrhage and the expression of TNF, IL-1ß, IL-18, and caspase 3. Finally, shock treatment had no adverse effect on behavioral recovery in contused rats pretreated with i.t. lidocaine. These observations suggest that i.t. lidocaine may provide a clinically safe method to counter the adverse effects of nociceptive input on tissue post-injury.

\section{Brain-dependent processes contribute to tissue loss and impaired recovery}

Our research has relied upon two model systems to explore spinal function: a transection paradigm used to examine plastic potential in the lumbosacral tissue in the absence of brain input and a contusion paradigm that has explored the implications of this work for recovery post-injury. The latter has focused on how nociceptive input affects tissue at the site of injury, rostral to the lumbosacral spinal cord. Our results suggest that noxious stimulation can enhance acute and chronic pain, undermine plastic potential, and impair recovery after a contusion injury. ${ }^{123,125}$ At the cellular level, we have uncovered some important similarities; both the induction of maladaptive plasticity in spinally transected rats and impaired recovery/ enhanced pain after a contusion injury are associated with a reduction in BDNF and increased expression of TNF. ${ }^{125,127}$ Likewise, the inhibition of adaptive learning has been linked to kappa opioid activity and research indicates that engaging this receptor system can undermine recovery after a contusion injury. ${ }^{68,150,151}$

Although the effects of nociceptive stimulation on lumbosacral function after spinal transection, and secondary injury after a contusion injury, are related, they are not identical. One difference is that the effect of uncontrollable/unpredictable shock on tissue sparing in contused rats is permanent whereas its effect on learning in spinally transected rats is reversible - it fades over time and the capacity for learning can be reinstated by a variety of treatments (e.g., i.t. BDNF, bicuculline, naltrexone, or sTNF). ${ }^{41,60,67,69,78}$ This reversibility mirrors the effect nociceptive stimulation has on adaptive plasticity/locomotor performance in the chronic phase of injury, after the period of secondary injury has passed. ${ }^{24-26}$

A second key difference concerns the initiation of these effects. In spinally transected rats, the induction of a learning impairment requires 6 min or more of VIS (180 shocks). ${ }^{41}$ In contrast, we have found that as little as $72 \mathrm{sec}$ of VIS (36 shocks) can affect the recovery process after a contusion injury. Indeed, even the irritation/inflammation associated with a peripheral cutaneous injection may have some effect on tissue sparing in contused rats. ${ }^{124}$ The fact that relatively little shock exposure induces hemorrhage, and thereby irreversibly harms spinal function, suggests that noxious stimulation will adversely affect spinal function in the acute stage of injury irrespective of whether it occurs in a regular or irregular manner. This is predicted because encoding temporal regularity 
within the lumbosacral spinal cord requires extended stimulus exposure (540 or more shocks), far more than that needed to induce hemorrhage. Here, behavioral control may be more effective because neural systems within the spinal cord seem "pre-wired" to detect R-O relations, ${ }^{120}$ which may serve to gate how a nociceptive stimulus is initially processed.

A final key difference stems from a recent discovery. At issue was whether spared fibers regulate the impact of nociceptive stimulation after a contusion injury. To explore this issue, Josh Reynolds contused the lower thoracic spinal cord as described above. ${ }^{152}$ Eighteen hours later, half the rats had the spinal cord transected at T2 whereas the remaining rats underwent a sham surgery. Six hours later, half the animals in each condition received VIS to the tail and tissue was collected $3 \mathrm{~h}$ later. As expected, VIS enhanced hemorrhage at the site of the contusion injury. To our surprise, this effect was abolished by a rostral transection. ${ }^{152}$ The results imply that the adverse effects of nociceptive stimulation at the site of injury involve additional, brain-dependent, processes.

To further explore the potential role of spared fibers, Anne Bopp applied lidocaine rostral to a contusion injury, before nociceptive stimulation. She found that this treatment attenuated the adverse effect of shock treatment. ${ }^{153}$ Likewise, inducing a state of surgical anesthesia before shock treatment had a protective effect. ${ }^{154}$ These observations imply that the acute effect of noxious stimulation on secondary injury depends, in some manner, upon communication with the brain. Given this, Kara Hudson examined whether noxious stimulation applied rostral to a contusion injury (to a forelimb) would affect recovery. Her preliminary results suggest that it does not. ${ }^{155}$ One interpretation of these findings is that the adverse effect of nociceptive stimulation on recovery depends upon two processes-nociceptive input caudal to injury and a brain-dependent system. We are currently exploring the mechanisms that contribute to the latter effect.

\section{Summary}

We have shown that spinal neurons, without input from the brain, can learn about causal $(\mathrm{R} \rightarrow \mathrm{O})$ and predictive $(\mathrm{S} \rightarrow \mathrm{S})$ relations and that this learning modulates how a noxious input affects spinal function. ${ }^{120}$ Our work shows that uncontrollable/unpredictable nociceptive stimulation induces a form of maladaptive plasticity that impairs spinal learning and enhances reactivity to mechanical stimulation. These effects were related to the induction of central sensitization and the upregulation of TNF. ${ }^{78,80,124} \mathrm{Ex}-$ posure to controllable/predictable stimulation fosters spinally mediated learning and has a lasting protective/restorative effect that counters the EMR and the learning impairment induced by both VIS and capsaicin. ${ }^{45,55,56}$ The beneficial effects of relational learning were linked to an upregulation of BDNF. ${ }^{60,120}$ These findings are consistent with other studies demonstrating that exercise, and locomotor training, enhance BDNF expression and can reduce behavioral signs of pain. ${ }^{156-159}$

Spinal injury places the spinal cord in a vulnerable state that enables the development of nociceptive sensitization. Injury appears to have this effect because it disrupts 5HT fibers within the DLF. ${ }^{86,96}$ The loss of this input leads to a downregulation of membrane-bound KCC2 and a loss of GABAergic inhibition. These changes can alter how GABAergic drugs impact spinal function. Further, in the presence of high intracellular $\mathrm{Cl}^{-}$concentrations, GABA can have a depolarizing effect that contributes to the development of nociceptive sensitization.

Noxious input impairs the recovery of locomotor function, and enhances the development of chronic pain, after a contusion injury. ${ }^{123,125}$ These effects are only observed if shock is given in an uncontrollable manner soon after injury and are related to a reduction in BDNF, and an increase in TNF, at the site of injury. ${ }^{127,125}$ Nociceptive input leads to increased tissue loss by increasing the extent of hemorrhage and apoptotic/pyroptotic cell death. Morphine treatment does not block these effects and has an adverse effect that increases tissue loss and mortality. ${ }^{146,148}$ Intrathecal lidocaine completely blocks the adverse effect of noxious input after a contusion injury and may provide an effective therapeutic treatment. ${ }^{149}$

How nociceptive stimulation affects spinal function post-injury depends upon a variety of factors, including spared fibers and time post-injury. ${ }^{123,152}$ Prolonged uncontrollable/unpredictable nociceptive stimulation caudal to a thoracic injury induces a state of maladaptive plasticity within the lumbosacral tissue that sensitizes nociceptive circuits and interferes with adaptive learning. ${ }^{46}$ These effects are most evident when injury disrupts communication with the brain and may continue to impact spinal function long after the initial insult. ${ }^{86}$ Less-severe uncontrollable noxious input soon after a contusion injury can have an additional effect that undermines long-term recovery by increasing the area of secondary injury. ${ }^{124}$ Here, a brain-dependent process appears to potentiate tissue loss. ${ }^{152}$

Our results show that nociceptive input can affect neural function in the absence of reported pain. Further, treatments that reduce psychological pain (e.g., morphine) can have unintended consequences that undermine long-term prognosis. In the acute phase of injury, treatments that inhibit the development of nociceptive sensitization (e.g., i.t. lidocaine) could promote tissue sparing and lower the incidence of chronic pain. Whereas the effects of nociceptive input on cell death wane over the weeks post-injury, uncontrollable/unpredictable input caudal to injury may continue to induce a state of maladaptive plasticity that undermines the beneficial effects of physical therapy and promotes pain. During the chronic phase of injury, presenting stimuli in a predictable/controllable manner could promote adaptive plasticity and attenuate the adverse consequences of noxious stimulation.

\section{Acknowledgments}

New findings and preparation of the manuscript were supported by grants from the Craig H. Neilsen Foundation (J.W.G., M.A.H., and R.M.M.), National Institute of Neurological Disorders and Stroke (NS0091723 [J.W.G., R.M.M.], NS081606 [S.M.G., M.A.H., and J.W.G.]), Mission Connect (J.W.G., M.A.H.), and the Mary Tucker Currie Professorship (J.W.G.).

We thank Josh Reynolds and Melissa Brumley for their contribution to this article. The synthesis presented here benefitted from discussions with Shawn Hochman, Edgar (Terry) Walters, David Magnuson, Jeffrey Petruska, and Alexander (Sasha) Rabchevsky.

\section{Author Disclosure Statement}

No competing financial interests exist.

\section{References}

1. Yang, Q., Wu, Z., Hadden, J.K., Odem, M.A., Zuo, Y., Crook, R.J., Frost, J.A., and Walters, E.T. (2014). Persistent pain after spinal cord injury is maintained by primary afferent activity. J. Neurosci. 34 , 10765-10769.

2. Fields, H.L. (2000). Pain modulation: expectation, opioid analgesia and virtual pain, in: Biological Basis for Mind Body Interactions. E.A. Mayer and C.B. Saper (eds). Elsevier: Amsterdam, pps. 245-253.

3. Basbaum, A.I. and Fields, H.L. (1984). Endogenous pain controlsystems - brain-stem spinal pathways and endorphin circuitry. Annu. Rev. Neurosci. 7, 309-338. 
4. Grau, J.W. (1987). Activation of the opioid and nonopioid analgesic systems-evidence for a memory hypothesis and against the coulometric hypothesis. J. Exp. Psychol. Anim. Behav. Process. 13, 215 225.

5. Fanselow, M.S. (1986). Conditioned fear-induced opiate analgesia: a competing motivational state theory of stress analgesia. Ann. N. Y. Acad. Sci. 467, 40-54.

6. Woolf, C.J. (1983). Evidence for a central component of post-injury pain hypersensitivity. Nature 306, 686-688.

7. Willis, W.D., Jr. (2001). Mechanisms of central sensitization of nociceptive dorsal horn neurons, in: Spinal Cord Plasticity: Alterations in Reflex Function. Springer-Verlag: New York, pps. 127-161.

8. Ji, R.R., Kohno, T., Moore, K.A., and Woolf, C.J. (2003). Central sensitization and LTP: do pain and memory share similar mechanisms? Trends Neurosci. 26, 696-705.

9. Latremoliere, A., and Woolf, C.J. (2009). Central sensitization: a generator of pain hypersensitivity by central neural plasticity. J. Pain 10, 895-926.

10. Lamotte, R.H., Shain, C.N., Simone, D.A., and Tsai, E.F.P. (1991). Neurogenic hyperalgesia-psychophysical studies of underlying mechanisms. J. Neurophysiol. 66, 190-211.

11. Simone, D.A., Baumann, T.K., and Lamotte, R.H. (1989). Dosedependent pain and mechanical hyperalgesia in humans after intradermal injection of capsaicin. Pain 38, 99-107.

12. Bear, M.F. (2003). Bidirectional synaptic plasticity: from theory to reality. Philos. Trans. R. Soc. Lond. B Biol. Sci. 358, 649-655.

13. Collingridge, G.L., and Bliss, T.V.P. (1987). NMDA receptorstheir role in long-term potentiation. Trends Neurosci. 10, 288-293.

14. Morris, R.G. (2013). NMDA receptors and memory encoding. Neuropharmacology 74, 32-40.

15. Dickenson, A.H., and Sullivan, A.F. (1987). Evidence for a role of the NMDA receptor in the frequency-dependent potentiation of deep rat dorsal horn nociceptive neurons following c-fiber stimulation. Neuropharmacology 26, 1235-1238.

16. Sandkuhler, J. (2000). Learning and memory in pain pathways. Pain $88,113-118$

17. Morris, R.G.M. (2013). NMDA receptors and memory encoding. Neuropharmacology 74, 32-40.

18. Watkins, L.R., Martin, D., Ulrich, P., Tracey, K.J., and Maier, S.F (1997). Evidence for the involvement of spinal cord glia in subcutaneous formalin induced hyperalgesia in the rat. Pain 71, 225-235.

19. Ji, R.R., Berta, T., and Nedergaard, M. (2013). Glia and pain: is chronic pain a gliopathy? Pain 154, Suppl. 1, S10-S28.

20. Huang, D., and Yu, B. (2010). The mirror-image pain: an unclered phenomenon and its possible mechanism. Neurosci. Biobehav. Rev. 34, 528-532.

21. Bardin, L., Schmidt, J., Alloui, A., and Eschalier, A. (2000). Effect of intrathecal administration of serotonin in chronic pain models in rats. Eur. J. Pharmacol. 409, 37-43.

22. Christensen, M.D., Everhart, A.W., Pickelman, J.T., and Hulsebosch, C.E. (1996). Mechanical and thermal allodynia in chronic central pain following spinal cord injury. Pain 68, 97-107.

23. Gwak, Y.S., and Hulsebosch, C.E. (2011). GABA and central neuropathic pain following spinal cord injury. Neuropharmacology 60 799-808.

24. Caudle, K.L., Brown, E.H., Shum-Siu, A., Burke, D.A., Magnuson, T.S.G., Voor, M.J., and Magnuson, D.S.K. (2011). Hindlimb immobilization in a wheelchair alters functional recovery following contusive spinal cord injury in the adult rat. Neurorehabil. Neural Repair 25, 729-739.

25. Caudle, K.L., Atkinson, D.A., Brown, E.H., Donaldson, K., Seibt, E. Chea, T., Smith, E., Chung, K., Shum-Siu, A., Cron, C.C., and Magnuson, D.S. (2015). Hindlimb stretching alters locomotor function after spinal cord injury in the adult rat. Neurorehabil. Neural Repair 29, 268-277.

26. Bouffard, J., Bouyer, L.J., Roy, J.S., and Mercier, C. (2014). Tonic pain experienced during locomotor training impairs retention despite normal performance during acquisition. J. Neurosci. 34, 9190-9195.

27. Grau, J.W. (2014). Learning from the spinal cord: how the study of spinal cord plasticity informs our view of learning. Neurobiol. Learn. Mem. 108, 155-171.

28. Grau, J.W., and Joynes, R.L. (2001). Pavlovian and instrumental conditioning within the spinal cord: methodological issues, in: Spinal Cord Plasticity: Alterations in Reflex Function. Kluwer: Boston, MA, pps. 13-54.
29. Grau, J.W., Salinas, J.A., Illich, P.A., and Meagher, M.W. (1990). Associative learning and memory for an antinociceptive response in the spinalized rat. Behav. Neurosci. 104, 489-494.

30. Domjan, M. (2015). Principles of Learning and Behavior. Cengage: Stamford, CT.

31. Grau, J.W. (2010). Instrumental conditioning, in: The Corsini Encyclopedia of Psychology. I.B. Weiner and W.E. Craighead (eds) John Wiley \& Sons: New York, pps. 480-481.

32. Grau, J.W., and Joynes, R.L. (2005). A neural-functionalist approach to learning. Int. J. Comp. Psychol. 18, 1-22.

33. Patterson, M.M. (2001). Classical conditioning of spinal reflexes: the first seventy years, in: Model Systems and the Neurobiology of Associative Learning. J.E. Steinmetz, M.A. Gluck, and P.R. Solomon (eds). Erlbaum: Mahwah, NJ, pps. 1-22.

34. Patterson, M.M., Cegavske, C.F., and Thompson, R.F. (1973). Effects of a classical-conditioning paradigm on hind-limb flexor nerve response in immobilized spinal cats. J. Comp. Physiol. Psychol. 84, 88-97.

35. Buerger, A.A., and Chopin, S.F. (1976). Instrumental avoidance conditioning in spinal vertebrates. Adv. Psychobiol. 3, 437-461.

36. Chopin, S.F., and Buerger, A.A. (1976). Instrumental avoidanceconditioning in spinal rat. Brain Res. Bull. 1, 177-183.

37. Church, R.M. (1964). Systematic effect of random error in the yoked control design. Psychol. Bull. 62, 122-131.

38. Church, R.M., and Lerner, N.D. (1976). Does headless roach learn to avoid. Physiol. Psychol. 4, 439-442.

39. Edgerton, V.R., Tillakaratne, N.J.K., Bigbee, A.J., de Leon, R.D., and Roy, R.R. (2004). Plasticity of the spinal neural circuitry after injury. Annu. Rev. Neurosci. 27, 145-167.

40. Grau, J.W., Barstow, D.G., and Joynes, R.L. (1998). Instrumental learning within the spinal cord: I. Behavioral properties. Behav. Neurosci. 112, 1366-1386.

41. Crown, E.D., Ferguson, A.R., Joynes, R.L., and Grau, J.W. (2002). Instrumental learning within the spinal cord: IV. Induction and retention of the behavioral deficit observed after noncontingent shock. Behav. Neurosci. 116, 1032-1051.

42. Joynes, R.L., Ferguson, A.R., Crown, E.D., Patton, B.C., and Grau, J.W. (2003). Instrumental learning within the spinal cord: V. Evidence the behavioral deficit observed after noncontingent nociceptive stimulation reflects an intraspinal modification. Behav. Brain Res. $141,159-170$

43. Maier, S.F., and Seligman, M.E.P. (1976). Learned helplessnesstheory and evidence. J. Exp. Psychol. Gen. 105, 3-46.

44. Hook, M.A., and Grau, J.W. (2007). An animal model of functional electrical stimulation: evidence that the central nervous system modulates the consequences of training. Spinal Cord 45, 702-712.

45. Hook, M.A., Huie, J.R., and Grau, J.W. (2008). Peripheral inflammation undermines the plasticity of the isolated spinal cord. Behav. Neurosci. 122, 233-249.

46. Ferguson, A.R., Crown, E.D., and Grau, J.W. (2006). Nociceptive plasticity inhibits adaptive learning in the spinal cord. Neuroscience 141, 421-431.

47. Ferguson, A.R., Huie, J.R., Crown, E.D., and Grau, J.W. (2012). Central nociceptive sensitization vs. spinal cord training: opposing forms of plasticity that dictate function after complete spinal cord injury. Front. Physiol. 3, 396.

48. Baumbauer, K.M., Hoy, K.C., Huie, J.R., Hughes, A.J., Woller, S.A., Puga, D.A., Setlow, B., and Grau, J.W. (2008). Timing in the absence of supraspinal input I: variable, but not fixed, spaced stimulation of the sciatic nerve undermines spinally-mediated instrumental learning. Neuroscience 155, 1030-1047.

49. Baumbauer, K.M., Young, E.E., Hoy, K.C., Jr., and Joynes, R.L. (2007). Neurokinin receptors modulate the impact of uncontrollable stimulation on adaptive spinal plasticity. Behav. Neurosci. 121, 1082-1094.

50. Malenka, R.C. (1994). Synaptic plasticity in the hippocampus: LTP and LTD. Cell 78, 535-538.

51. Mauk, M.D. and Buonomano, D.V. (2004). The neural basis of temporal processing. Annu. Rev. Neurosci. 27, 307-340.

52. Baumbauer, K.M., Huie, J.R., Hughes, A.J., and Grau, J.W. (2009). Timing in the absence of supraspinal input II: regularly spaced stimulation induces a lasting alteration in spinal function that depends on the NMDA receptor, BDNF release, and protein synthesis. J. Neurosci. 29, 14383-14393.

53. Lee, K.H., Turtle, J. D., Strain, M. M., Huang, Y.-J., Baumbauer, K. M., and Grau, J. W. (2015). Learning about time within the spinal 
cord: Evidence that spinal neurons can abstract and store an index of regularity. Front. Behav. Neurosci. 9, 274.

54. Lee, K.H., Huang, Y.-J., and Grau, J. W. (2016). Learning about time within the spinal cord II: Evidence that temporal regularity is encoded by a spinal oscillator. Front. Behav. Neurosci. 10, 14.

55. Baumbauer, K.M., and Grau, J.W. (2011). Timing in the absence of supraspinal input III: regularly spaced cutaneous stimulation prevents and reverses the spinal learning deficit produced by peripheral inflammation. Behav. Neurosci. 125, 37-45.

56. Baumbauer, K.M., Lee, K.H., Puga, D.A., Woller, S.A., Hughes, A.J., and Grau, J.W. (2012). Temporal regularity determines the impact of electrical stimulation on tactile reactivity and response to capsaicin in spinally transected rats. Neuroscience 227, 119-133.

57. Groves, P.M., and Thompson, R.F. (1970). Habituation a dual process theory. Psychol. Rev. 77, 419-450.

58. Joynes, R.L., Janjua, K., and Grau, J.W. (2004). Instrumental learning within the spinal cord: VI. The NMDA receptor antagonist, AP5, disrupts the acquisition and maintenance of an acquired flexion response. Behav. Brain Res. 154, 431-438.

59. Gomez-Pinilla, F., Huie, J.R., Ying, Z., Ferguson, A.R., Crown, E.D. Baumbauer, K.M., Edgerton, V.R., and Grau, J.W. (2007). BDNF and learning: Evidence that instrumental training promotes learning within the spinal cord by up-regulating BDNF expression. Neuroscience 148, 893-906.

60. Huie, J.R., Garraway, S.M., Baumbauer, K.M., Hoy, K.C., Beas, B.S., Montgomery, K.S., Bizon, J.L., and Grau, J.W. (2012). Brainderived neurotrophic factor promotes adaptive plasticity within the spinal cord and mediates the beneficial effects of controllable stimulation. Neuroscience 200, 74-90.

61. Grau, J.W., Huie, J.R., Lee, K.H., Hoy, K.C., Huang, Y.J., Turtle, J.D. Strain, M.M., Baumbauer, K.M., Miranda, R.M., Hook, M.A., Ferguson, A.R., and Garraway, S.M. (2014). Metaplasticity and behavior: how training and inflammation affect plastic potential within the spinal cord and recovery after injury. Front. Neural Circuits 8, 100.

62. Abraham, W.C., and Bear, M.F. (1996). Metaplasticity: the plasticity of synaptic plasticity. Trends Neurosci. 19, 126-130.

63. Hawkins, R.D., Kandel, E.R., and Bailey, C.H. (2006). Molecula mechanisms of memory storage in Aplysia. Biol. Bull. 210, 174-191.

64. Maier, S.F., and Watkins, L.R. (2005). Stressor controllability and learned helplessness: the roles of the dorsal raphe nucleus, serotonin, and corticotropin-releasing factor. Neurosci. Biobehav. Rev. 29, 829-841.

65. Meagher, M.W., Grau, J.W., and King, R.A. (1990). Role of supraspinal systems in environmentally induced antinociception-effect of spinalization and decerebration on brief shock-induced and long shock-induced antinociception. Behav. Neurosci. 104, 328-338.

66. Watkins, L.R., Cobelli, D.A., and Mayer, D.J. (1982). Opiate vs nonopiate footshock induced analgesia (FSIA) - descending and intraspinal components. Brain Res. 245, 97-106.

67. Joynes, R.L., and Grau, J.W. (2004). Instrumental learning within the spinal cord: III. Prior exposure to noncontingent shock induces a behavioral deficit that is blocked by an opioid antagonist. Neurobiol. Learn. Mem. 82, 35-51.

68. Washburn, S.N., Maultsby, M.L., Puga, D.A., and Grau, J.W. (2008) Opioid regulation of spinal cord plasticity: evidence the kappa-2 opioid receptor agonist GR89696 inhibits learning within the rat spinal cord. Neurobiol. Learn. Mem. 89, 1-16.

69. Ferguson, A.R., Washburn, S.N., Crown, E.D., and Grau, J.W. (2003). GABA(A) receptor activation is involved in noncontingent shock inhibition of instrumental conditioning in spinal rats. Behav. Neurosci. 117, 799-812.

70. Meagher, M.W., Chen, P.S., Salinas, J.A., and Grau, J.W. (1993) Activation of the opioid and nonopioid hypoalgesic systems at the level of the brain-stem and spinal-cord-does a coulometric relation predict the emergence or form of environmentally induced hypoalgesia. Behav. Neurosci. 107, 493-505.

71. Moser, E.I., and Moser, M.B. (1999). Is learning blocked by saturation of synaptic weights in the hippocampus? Neurosci. Biobehav. Rev. 23, 661-672.

72. Vichaya, E.G., Baumbauer, K.M., Carcoba, L.M., Grau, J.W., and Meagher, M.W. (2009). Spinal glia modulate both adaptive and pathological processes. Brain Behav. Immun. 23, 969-976.

73. Young, E.E., Baumbauer, K.M., Elliot, A., and Joynes, R.L. (2007) Lipopolysaccharide induces a spinal learning deficit that is blocked by $\mathrm{IL}-1$ receptor antagonism. Brain Behav. Immun. 21, 748-757.
74. Beattie, E.C., Stellwagen, D., Morishita, W., Bresnahan, J.C., Ha, B.K., Von Zastrow, M., Beattie, M.S., and Malenka, R.C. (2002). Control of synaptic strength by glial TNF alpha. Science 295, 22822285 .

75. Stellwagen, D., and Malenka, R.C. (2006). Synaptic scaling mediated by glial TNF-alpha. Nature 440, 1054-1059.

76. Czeschik, J.C., Hagenacker, T., Schaefers, M., and Buesselberg, D. (2008). TNF-alpha differentially modulates ion channels of nociceptive neurons. Neurosci. Lett. 434, 293-298.

77. Park, C.-K., Lue, N., Xu, Z.-Z., Liu, T., Serhan, C.N., and Ji, R.-R. (2011). Resolving TRPV1-and TNF-alpha-mediated spinal cord synaptic plasticity and inflammatory pain with neuroprotectin D1. J. Neurosci. 31, 15072-15085.

78. Huie, J.R., Baumbauer, K.M., Lee, K.H., Bresnahan, J.C., Beattie, M.S., Ferguson, A.R., and Grau, J.W. (2012). Glial tumor necrosis factor alpha (TNF alpha) generates metaplastic inhibition of spinal learning. PLoS One 7, e39751.

79. Crown, E.D., and Grau, J.W. (2001). Preserving and restoring behavioral potential within the spinal cord using an instrumental training paradigm. J. Neurophysiol. 86, 845-855.

80. Ferguson, A.R., Huie, J.R., Crown, E.D., Baumbauer, K.M., Hook, M.A., Garraway, S.M., Lee, K.H., Hoy, K.C., and Grau, J.W. (2012). Maladaptive spinal plasticity opposes spinal learning and recovery in spinal cord injury. Front. Physiol. 3, 399.

81. Eaton, M.J., Santiago, D.I., Dancausse, H.A., and Whittemore, S.R. (1997). Lumbar transplants of immortalized serotonergic neurons alleviate chronic neuropathic pain. Pain 72, 59-69.

82. Hains, B.C., Fullwood, S.D., Eaton, M.J., and Hulsebosch, C.E. (2001). Subdural engraftment of serotonergic neurons following spinal hemisection restores spinal serotonin, downregulates serotonin transporter, and increases BDNF tissue content in rat. Brain Res. 913 $35-46$.

83. Hains, B.C., Johnson, K.M., McAdoo, D.J., Eaton, M.J., and Hulsebosch, C.E. (2001). Engraftment of serotonergic precursors enhances locomotor function and attenuates chronic central pain behavior following spinal hemisection injury in the rat. Exp. Neurol. $171,361-378$

84. Hains, B.C., Willis, W.D., and Hulsebosch, C.E. (2003). Serotonin receptors 5-HT1A and 5-HT3 reduce hyperexcitability of dorsal horn neurons after chronic spinal cord hemisection injury in rat. Exp. Brain Res. 149, 174-186.

85. Gjerstad, J., Tjolsen, A., and Hole, K. (2001). Induction of long-term potentiation of single wide dynamic range neurones in the dorsal horn is inhibited by descending pathways. Pain 91, 263-268.

86. Crown, E.D., and Gran, J.W. (2005). Evidence that descending serotonergic systems protect spinal cord plasticity against the disruptive effect of uncontrollable stimulation. Exp. Neurol. 196, 164-176.

87. Davies, J.E., Marsden, C.A., and Roberts, M.H. (1983). Hyperalgesia and the reduction of monoamines resulting from lesions of the dorsolateral funiculus. Brain Res. 261, 59-68.

88. Brustein, E., and Rossignol, S. (1999). Recovery of locomotion after ventral and ventrolateral spinal lesions in the cat. II. Effects of noradrenergic and serotoninergic drugs. J. Neurophysiol. 81, 15131530 .

89. Rossignol, S., Chau, C., Brustein, E., Giroux, N., Bouyer, L., Barbeau, H., and Reader, T.A. (1998). Pharmacological activation and modulation of the central pattern generator for locomotion in the cat. Ann. N. Y. Acad. Sci. 860, 346-359.

90. Baba, H., Ji, R.R., Kohno, T., Moore, K.A., Ataka, T., Wakai, A., Okamoto, M., and Woolf, C.J. (2003). Removal of GABAergic inhibition facilitates polysynaptic A fiber-mediated excitatory transmission to the superficial spinal dorsal horn. Mol. Cell. Neurosci. 24 818-830.

91. Sorkin, L.S., Puig, S., and Jones, D.L. (1998). Spinal bicuculline produces hypersensitivity of dorsal horn neurons: effects of excitatory amino acid antagonists. Pain 77, 181-190.

92. Zhang, Z., Hefferan, M.P., and Loomis, C.W. (2001). Topical bicuculline to the rat spinal cord induces highly localized allodynia that is mediated by spinal prostaglandins. Pain 92, 351-361.

93. Sivilotti, L., and Woolf, C.J. (1994). The contribution of GABAA and glycine receptors to central sensitization: disinhibition and touchevoked allodynia in the spinal cord. J. Neurophysiol. 72, 169-179.

94. Dougherty, K.J., and Hochman, S. (2008). Spinal cord injury causes plasticity in a subpopulation of lamina I GABAergic interneurons. J. Neurophysiol. 100, 212-223. 
95. Huang, Y.-J., Lee, K.H., Garraway, S.M., and Grau, J. W. (2013). SCI alters the effect of GABAergic system on nociception: different effect of GABA-A antagonist bicuculline on capsaicin-indued allodynia in sham vs. spinally transected rats. 2013 Neuroscience Meeting Planner. Society for Neuroscience: San Diego, CA.

96. Huang, Y.-J., Lee, K. H., Murphy, L., Garraway, S. M., and Grau, J. W. (2016). Spinal cord injury (SCI) transforms how GABA affects nociceptive plasticity. Exp. Neurol. 288, 38-50.

97. Cramer, S.W., Baggott, C., Cain, J., Tilghman, J., Allcock, B., Miranpuri, G., Rajpal, S., Sun, D., and Resnick, D. (2008). The role of cation-dependent chloride transporters in neuropathic pain following spinal cord injury. Mol. Pain 4, 36.

98. Ben-Ari, Y. (2002). Excitatory actions of GABA during development: the nature of the nurture. Nat. Rev. Neurosci. 3, 728-739.

99. Ben-Ari, Y., Khalilov, I., Kahle, K.T., and Cherubini, E. (2012). The GABA excitatory/inhibitory shift in brain maturation and neurological disorders. Neuroscientist 18, 467-486.

100. Drew, G.M., Siddall, P.J., and Duggan, A.W. (2004). Mechanical allodynia following contusion injury of the rat spinal cord is associated with loss of GABAergic inhibition in the dorsal horn. Pain 109, 379-388.

101. Boulenguez, P., Liabeuf, S., Bos, R., Bras, H., Jean-Xavier, C., Brocard, C., Stil, A., Darbon, P., Cattaert, D., Delpire, E., Marsala, M., and Vinay, L. (2010). Down-regulation of the potassium-chloride cotransporter KCC2 contributes to spasticity after spinal cord injury. Nat. Med. 16, 302-307.

102. Hasbargen, T., Ahmed, M.M., Miranpuri, G., Li, L., Kahle, K.T., Resnick, D., and Sun, D. (2010). Role of NKCC1 and KCC2 in the development of chronic neuropathic pain following spinal cord injury. Ann. N. Y. Acad. Sci. 1198, 168-172.

103. Lu, Y., Zheng, J., Xiong, L., Zimmermann, M., and Yang, J. (2008). Spinal cord injury-induced attenuation of GABAergic inhibition in spinal dorsal horn circuits is associated with down-regulation of the chloride transporter KCC2 in rat. J. Physiol. 586, 5701-5715.

104. Huang, Y.-J., Lee, K.H., and Grau, J. W. (2014). Spinal injury transforms how GABAergic system regulate nociceptive systems within the spinal cord: role of KCC2. 2014 Neuroscience Meeting Planner. Society for Neuroscience: Washington, DC.

105. Huang, Y.-J., and Grau, J. W. (2012). Effect of GABA-A antagonist bicuculline and GABA-A agonist muscimol on the allodynia induced by capsaicin in spinal transected rats. 2012 Neuroscience Meeting Planner. Society for Neuroscience: New Orleans, LA.

106. Young, E.E., Baumbauer, K.M., Elliot, A., and Joynes, R.L. (2007). Neonatal hind-paw injury disrupts acquisition of an instrumental response in adult spinal rats. Behav. Neurosci. 121, 1095-1100.

107. Baumbauer, K.M., Young, E.E., and Joynes, R.L. (2009). Pain and learning in a spinal system: contradictory outcomes from common origins. Brain Res. Rev. 61, 124-143.

108. Huang, Y.-J., Lee, K. H., and Grau, J. W. (2015). Loss of descending serotonergic fibers transforms how GABA regulates nociceptive systems within the spinal cord: role of KCC2. 2015 Neuroscience Meeting Planner. Society for Neuroscience: Chicago, IL.

109. Huang, Y.-J., and Grau, J. W. (submitted). Descending serotongeric fibers regulate nociceptive sensitization through KCC2 after spinal injury.

110. King, T., Vera-Portocarrero, L., Gutierrez, T., Vanderah, T.W., Dussor, G., Lai, J., Fields, H.L., and Porreca, F. (2009). Unmasking the tonicaversive state in neuropathic pain. Nat. Neurosci. 12, 1364-1366.

111. Lee, K.H., Baumbauer, K.M., and Grau, J.W. (2014). Brain-derived neurotrophic factor reduces inflammation-induced allodynia. 2014 Neuroscience Meeting Planner. Society for Neuroscience: Washington, DC.

112. Coull, J.A., Beggs, S., Boudreau, D., Boivin, D., Tsuda, M., Inoue, K., Gravel, C., Salter, M.W., and De Koninck, Y. (2005). BDNF from microglia causes the shift in neuronal anion gradient underlying neuropathic pain. Nature 438, 1017-1021

113. Valencia-de Ita, S., Lawand, N.B., Lin, Q., Castaneda-Hernandez, G., and Willis, W.D. (2006). Role of the Na+-K+-2Cl- cotransporter in the development of capsaicin-induced neurogenic inflammation. $\mathrm{J}$. Neurophysiol. 95, 3553-3561.

114. Lu, B., Pang, P.T., and Woo, N.H. (2005). The yin and yang of neurotrophin action. Nat. Rev. Neurosci. 6, 603-614.

115. Biggs, J.E., Lu, V.B., Stebbing, M.J., Balasubramanyan, S., and Smith, P.A. (2010). Is BDNF sufficient for information transfer between microglia and dorsal horn neurons during the onset of central sensitization? Mol. Pain 6, 44 .
116. Huang, Y.J., Lee, K. H., and Grau, J. W. (2016). Ying-yang of BDNF: $\mathrm{KCC} 2$ expressional change regulate the effect of BDNF before and after spinal cord injury (SCI). An effective treatment for reducing secondary injury after SCI and acute pain. 2016 Neuroscience Meeting Planner. Society for Neuroscience: San Diego, CA.

117. Jolivalt, C.G., Lee, C.A., Ramos, K.M.,, and Calcutt, N.A. (2008). Allodynia and hyperalgesia in diabetic rats are mediated by GABA and depletion of spinal potassium-chloride co-transporters. Pain 140, 48-57.

118. Rivera, C., Voipio, J., Thomas-Crusells, J., Li, H., Emri, Z., Sipila, S., Payne, J.A., Minichiello, L., Saarma, M., and Kaila, K. (2004). Mechanism of activity-dependent downregulation of the neuronspecific K-Cl cotransporter KCC2. J. Neurosci. 24, 4683-4691.

119. Tashiro, S., Shinozaki, M., Mukaino, M., Renault-Mihara, F., Toyama, Y., Liu, M., Nakamura, M., and Okano, H. (2015). BDNF induced by treadmill training contributes to the suppression of spasticity and allodynia after spinal cord injury via upregulation of KCC2. Neurorehabil. Neural Repair 29, 677-689.

120. Grau, J.W., Huie, J.R., Garraway, S.M., Hook, M.A., Crown, E.D., Baumbauer, K.M., Lee, K.H., Hoy, K.C., and Ferguson, A.R. (2012). Impact of behavioral control on the processing of nociceptive stimulation. Front. Physiol. 3, 262.

121. Grau, J.W. (2006). Spinal neurons exhibit a surprising capacity to learn and a hidden vulnerability when freed from the brain's control. Curr. Neurol. Neurosci. Rep. 6, 177-180.

122. Harkema, S., Gerasimenko, Y., Hodes, J., Burdick, J., Angeli, C., Chen, Y., Ferreira, C., Willhite, A., Rejc, E., Grossman, R.G., and Edgerton, V.R. (2011). Effect of epidural stimulation of the lumbosacral spinal cord on voluntary movement, standing, and assisted stepping after motor complete paraplegia: a case study. Lancet 377 , 1938-1947.

123. Washburn, S.N., Hook, M.A., Ferguson, A.R., Crown, E.D., Garcia, G., Bolding, K.A., Miranda, R.C., and Grau, J.W. (2004). Uncontrollable stimulation undermines recovery of function after spinal cord injury. J. Neurotrauma 21, 1291-1291.

124. Grau, J.W., Strain, M.M., Huang, Y.-J., Turtle, J.D., Lee, K.H., Kozicz, A., Pesek, B.S., and Garraway, S.M. (2014). Peripheral inflammation disrupts locomotor recovery after spinal contusion injury. 2014 Neuroscience Meeting Planner. Society for Neuroscience: Washington, DC.

125. Garraway, S.M., Woller, S.A., Huie, J.R., Hartman, J.J., Hook, M.A Miranda, R.C., Huang, Y.-J., Ferguson, A.R., and Grau, J.W. (2014). Peripheral noxious stimulation reduces withdrawal threshold to mechanical stimuli after spinal cord injury: role of tumor necrosis factor alpha and apoptosis. Pain 155, 2344-2359.

126. Coderre, T.J., Katz, J., Vaccarino, A.L., and Melzack, R. (1993) Contribution of central neuroplasticity to pathological pain-review of clinical and experimental evidence. Pain 52, 259-285.

127. Garraway, S.M., Turtle, J.D., Huie, J.R., Lee, K.H., Hook, M.A., Woller, S.A., and Grau, J.W. (2011). Intermittent noxious stimulation following spinal cord contusion injury impairs locomotor recovery and reduces spinal brain-derived neurotrophic factor-tropomyosinreceptor kinase signaling in adult rats. Neuroscience 199, 86-102.

128. Xu, J., E, X., Liu, H., Li, F., Cao, Y., Tian, J., and Yan, J. (2015). Tumor necrosis factor-alpha is a potential diagnostic biomarker for chronic neuropathic pain after spinal cord injury. Neurosci. Lett. 595 30-34.

129. Grell, M., Douni, E., Wajant, H., Lohden, M., Clauss, M., Maxeiner, B., Georgopoulos, S., Lesslauer, W., Kollias, G., Pfizenmaier, K., and Scheurich, P. (1995). The transmembrane form of tumor necrosis factor is the prime activating ligand of the $80 \mathrm{kDa}$ tumor necrosis factor receptor. Cell 83, 793-802.

130. Santee, S.M., and Owen-Schaub, L.B. (1996). Human tumor necrosis factor receptor p75/80 (CD120b) gene structure and promoter characterization. J. Biol. Chem. 271, 21151-21159.

131. Yan, P., Liu, N., Kim, G.M., Xu, J., Xu, J., Li, Q., Hsu, C.Y., and Xu, X.M. (2003). Expression of the type 1 and type 2 receptors for tumor necrosis factor after traumatic spinal cord injury in adult rats. Exp. Neurol. 183, 286-297.

132. Bethea, J.R. (2000). Spinal cord injury-induced inflammation: a dualedged sword. Prog. Brain Res. 128, 33-42.

133. Zhang, L., Berta, T., Xu, Z.-Z., Liu, T., Park, J.Y., and Ji, R.-R. (2011). TNF-alpha contributes to spinal cord synaptic plasticity and inflammatory pain: distinct role of TNF receptor subtypes 1 and 2 . Pain 152, 419-427. 
134. Huie, J.R., Stuck, E.D., Lee, K.H., Irvine, K.A., Beattie, M.S., Bresnahan, J.C., Grau, J.W., and Ferguson, A. R. (2015). AMPA receptor phosphorylation and synaptic co-localization on motor neurons drive maladaptive plasticity below complete spinal cord injury. eNeuro 2, ENEURO.0091-15.2015. Published online 2015 Nov 16. Prepublished online 2015 Oct 30. doi: 10.1523/ENEURO.0091-15.2015

135. Hsu, H.L., Shu, H.B., Pan, M.G., and Goeddel, D.V. (1996). TRADD-TRAF2 and TRADD-FADD interactions define two distinct TNF receptor 1 signal transduction pathways. Cell 84, 299-308.

136. Shu, H.B., Takeuchi, M., and Goeddel, D.V. (1996). The tumor necrosis factor receptor 2 signal transducers TRAF2 and c-IAP1 are components of the tumor necrosis factor receptor 1 signaling complex. Proc. Natl. Acad. Sci. U. S. A. 93, 13973-13978.

137. Lee, Y.B., Yune, T.Y., Baik, S.Y., Shin, Y.H., Du, S., Rhim, H., Lee, E.B., Kim, Y.C., Shin, M.L., Markelonis, G.J., and Oh, T.H. (2000). Role of tumor necrosis factor-alpha in neuronal and glial apoptosis after spinal cord injury. Exp. Neurol. 166, 190-195.

138. de Rivero Vaccari, J.P., Dietrich, W.D., and Keane, R.W. (2016) Therapeutics targeting the inflammasome after central nervous system injury. Transl. res. 167, 35-45.

139. de Rivero Vaccari, J.P., Lotocki, G., Alonso, O.F., Bramlett, H.M., Dietrich, W.D., and Keane, R.W. (2009). Therapeutic neutralization of the NLRP1 inflammasome reduces the innate immune response and improves histopathology after traumatic brain injury. J. Cereb. Blood Flow Metab. 29, 1251-1261.

140. de Rivero Vaccari, J.P., Lotocki, G., Marcillo, A.E., Dietrich, W.D., and Keane, R.W. (2008). A molecular platform in neurons regulates inflammation after spinal cord injury. J. Neurosci. 28, 3404-3414.

141. Turtle, J., Strain, M., Reynolds, J., Huang, Y.-J., Garraway, S., and Grau, J. (2014). Nociceptive stimulation induces caspase 1 activation following spinal cord injury. J. Neurotrauma 33, A-109.

142. Brumley, M.K., Turtle, J., Forsberg, J., Reynolds, J., Huang, Y.-J., and Grau, J. W. (2016). Peripheral pain increases lesion-site hemorrhage after contusive spinal cord injury. J. Neurotrauma 33, A-108.

143. Simard, J.M., Popovich, P.G., Tsymbalyuk, O., Caridi, J., Gullapalli, R.P., Kilbourne, M.J., and Gerzanich, V. (2013). MRI evidence that glibenclamide reduces acute lesion expansion in a rat model of spinal cord injury. Spinal Cord 51, 823-827.

144. Brumley, M.K., Turtle, J. D., Forsberg, J. M., and Grau, J. W. (2016). Acute pain after SCI exacerbates progressive hemorrhagic necrosis 2016 Neuroscience Meeting Planner. Society for Neuroscience: San Diego, CA.

145. Beggs, S., Liu, X.J., Kwan, C., and Salter, M.W. (2010). Peripheral nerve injury and TRPV1-expressing primary afferent $\mathrm{C}$-fibers cause opening of the blood-brain barrier. Mol. Pain 6, 74

146. Hook, M.A., Liu, G.T., Washburn, S.N., Ferguson, A.R., Bopp, A.C., Huie, J.R., and Grau, J.W. (2007). The impact of morphine after a spinal cord injury. Behav. Brain Res. 179, 281-293.

147. Hook, M.A., Moreno, G., Woller, S., Puga, D., Hoy, K., Balden, R., and Grau, J.W. (2009). Intrathecal morphine attenuates recovery of function after a spinal cord injury. J. Neurotrauma 26, 741-752.

148. Hook, M.A., Washburn, S.N., Moreno, G., Woller, S.A., Puga, D., Lee, K.H., and Grau, J.W. (2011). An IL-1 receptor antagonist blocks a morphine-induced attenuation of locomotor recovery after spinal cord injury. Brain Behav. Immun. 25, 349-359.

149. Strain, M.M., Turtle, J. D., Huang, Y-. J., Reynolds, J. A., Brumley, M. K., DeLeon, A., and Grau, J. W. (2016). Lidocaine blocks the detrimental effects of C-fiber activation on recovery after injury. $\mathrm{J}$. Neurotrauma 33, A-72.

150. Faden, A.I. (1990). Opioid and nonopioid mechanisms may contribute to dynorphins pathophysiological actions in spinal-cord injury. Ann. Neurol. 27, 67-74.

151. Aceves, M., Mathai, B.B., and Hook, M.A. (2016). Evaluation of the effects of specific opioid receptor agonists in a rodent model of spinal cord injury. Spinal Cord 54, 767-777.

152. Reynolds, J.A., Turtle, J. D., Huang, Y.J., Strain, M. M., and Grau, J. W. (2016). Spared fibers promote the development of secondary spinal injury in response to acute pain. 2016 Neuroscience Meeting Planner. Society for Neuroscience: San Diego, CA.

153. Bopp, A.C., Ferguson, A.R., Miranda, R.C., and Grau, J.W. (2003). Blocking neural conduction rostral to an injury in rats has a protective effect that reduces the adverse consequences of nociceptive stimulation. 2003 Abstract Viewer/Itinerary Planner. Society for Neuroscience: Washington, DC.

154. Bopp, A.C., and Grau, J.W. (2004). Brain-dependent processes contribute to the adverse consequences of nociceptive stimulation on recovery of function. J. Neurotrauma 21, 1290.

155. Hudson, K.L. (2009). Intermitten stimulation below, but not above, a moderate contusion injury impairs the recovery of locomotor function in rats. 2009 Neuroscience Meeting Planner. Society for Neuroscience: Chicago, IL.

156. Gomez-Pinilla, F., Ying, Z., Opazo, P., Roy, R.R., and Edgerton, V.R. (2001). Differential regulation by exercise of BDNF and NT-3 in rat spinal cord and skeletal muscle. Eur. J. Neurosci. 13, 1078-1084.

157. Gomez-Pinilla, F., Ying, Z., Roy, R.R., Molteni, R., and Edgerton, V.R. (2002). Voluntary exercise induces a BDNF-mediated mechanism that promotes neuroplasticity. J. Neurophysiol. 88, 2187-2195.

158. Hutchinson, K.J., Gomez-Pinilla, F., Crowe, M.J., Ying, Z., and Basso, D.M. (2004). Three exercise paradigms differentially improve sensory recovery after spinal cord contusion in rats. Brain 127, 14031414.

159. Dugan, E.A., and Sagen, J. (2015). An intensive locomotor training paradigm improves neuropathic pain following spinal cord compression injury in rats. J. Neurotrauma 32, 622-632.

Address correspondence to: James W. Grau, PhD

Behavioral and Cellular Neuroscience Department of Psychology Texas A\&M University

College Station, TX 77843-4235

E-mail: j-grau@tamu.edu 